\title{
Production of $\mathrm{K}^{*}(892)^{0}$ and $\phi(1020)$ in $\mathrm{p}-\mathrm{Pb}$ collisions at $\sqrt{s_{\mathrm{NN}}}=5.02 \mathrm{TeV}$
}

\author{
ALICE Collaboration * \\ CERN, 1211 Geneva 23, Switzerland
}

Received: 1 February 2016 / Accepted: 14 April 2016 / Published online: 30 April 2016

(C) CERN for the benefit of the ALICE collaboration 2016. This article is published with open access at Springerlink.com

\begin{abstract}
The production of $\mathrm{K}^{*}(892)^{0}$ and $\phi(1020)$ mesons has been measured in $\mathrm{p}-\mathrm{Pb}$ collisions at $\sqrt{s_{\mathrm{NN}}}=5.02 \mathrm{TeV}$. $\mathrm{K}^{* 0}$ and $\phi$ are reconstructed via their decay into charged hadrons with the ALICE detector in the rapidity range $-0.5<y<0$. The transverse momentum spectra, measured as a function of the multiplicity, have a $\mathrm{p}_{\mathrm{T}}$ range from 0 to $15 \mathrm{GeV} / c$ for $\mathrm{K}^{* 0}$ and from 0.3 to $21 \mathrm{GeV} / c$ for $\phi$. Integrated yields, mean transverse momenta and particle ratios are reported and compared with results in pp collisions at $\sqrt{s}$ $=7 \mathrm{TeV}$ and $\mathrm{Pb}-\mathrm{Pb}$ collisions at $\sqrt{s_{\mathrm{NN}}}=2.76 \mathrm{TeV}$. In $\mathrm{Pb}-\mathrm{Pb}$ and $\mathrm{p}-\mathrm{Pb}$ collisions, $\mathrm{K}^{* 0}$ and $\phi$ probe the hadronic phase of the system and contribute to the study of particle formation mechanisms by comparison with other identified hadrons. For this purpose, the mean transverse momenta and the differential proton-to- $\phi$ ratio are discussed as a function of the multiplicity of the event. The short-lived $\mathrm{K}^{* 0}$ is measured to investigate re-scattering effects, believed to be related to the size of the system and to the lifetime of the hadronic phase.
\end{abstract}

\section{Introduction}

The phase transition predicted by QCD from ordinary matter to a deconfined quark-gluon plasma (QGP) has been studied in high-energy heavy-ion collision (AA) experiments at the super proton synchrotron (SPS) [1-11], the relativistic heavy-ion collider (RHIC) [12-15] and the large hadron collider (LHC) [16-22]. In this context, hadronic resonances provide an important contribution to the study of particle production mechanisms and the characterisation of the dynamic evolution of the system formed in heavy-ion collisions, during the late hadronic phase. Results on resonance production in different collision systems at RHIC have been reported in

See Acknowledgments section for the list of collaboration members.

^e-mail: alice-publications@cern.ch
[23-29]. At the $\mathrm{LHC}, \mathrm{K}^{*}(892)^{0}$ and $\phi(1020)$ production have been measured in pp collisions at $\sqrt{s}=7 \mathrm{TeV}$ by ALICE [30], ATLAS [31] and LHCb [32], and in pp and Pb-Pb collisions at $\sqrt{s_{\mathrm{NN}}}=2.76 \mathrm{TeV}$ by ALICE [33,34]. Results obtained in $\mathrm{p}-\mathrm{Pb}$ collisions at $\sqrt{s_{\mathrm{NN}}}=5.02 \mathrm{TeV}$ with the ALICE detector are presented in this paper.

Measurements in smaller collision systems such as proton-proton ( $\mathrm{pp}$ ) and proton-nucleus ( $\mathrm{pA}$ ) constitute a reference for the interpretation of the heavy-ion results. In addition, proton-nucleus collisions have proven to be interesting in their own right, as several measurements [35-39] indicate that they cannot be explained by an incoherent superposition of pp collisions, but suggest instead the presence of collective effects [40,41]. In heavy-ion collisions, the presence of a strong collective radial flow reveals itself in the evolution with centrality of the transverse momentum spectra of identified hadrons [42]. The spectral shapes of $\mathrm{K}^{* 0}$ and $\phi$ follow the common behaviour found for all the other particles and exhibit an increase of the mean transverse momentum, dominated by the low $p_{\mathrm{T}}$ region of the spectra where particle production is more abundant, with centrality [33]. In central $\mathrm{Pb}-\mathrm{Pb}$ events, particles with similar mass such as the $\phi$ meson and the proton have similar $\left\langle p_{\mathrm{T}}\right\rangle$ and, in addition, the $\phi / \mathrm{p}$ ratio as a function of $p_{\mathrm{T}}$ is flat for $p_{\mathrm{T}}<4 \mathrm{GeV} / c$. Both observations are consistent with expectations from hydrodynamic models, where the mass of the particle drives the particle spectral shapes at low momenta [43]. On the other hand, in most peripheral $\mathrm{Pb}-\mathrm{Pb}$ collisions, as well as in $\mathrm{pp}$, the $\phi / \mathrm{p}$ ratio exhibits a strong $p_{\mathrm{T}}$ dependence, suggesting that the production of lowand intermediate-momentum baryons and mesons occurs by means of other mechanisms such as fragmentation or recombination $[44,45]$.

Similarly to $\mathrm{Pb}-\mathrm{Pb}$, one is interested in searching for collective effects in $\mathrm{p}-\mathrm{Pb}$ collisions and in studying particle production as a function of the hadron multiplicity, which strongly depends on the geometry of the collision. In this respect, $\mathrm{p}-\mathrm{Pb}$ collisions provide us with a system whose size in terms of average charged-particle density and num- 
ber of participating nucleons is intermediate between pp and peripheral $\mathrm{Pb}-\mathrm{Pb}$ collisions [18,46-49]. Measurements in an intermediate-size system as $\mathrm{p}-\mathrm{Pb}$ can provide information on the onset of the collective behaviour leading to the presence of radial flow.

The $\phi$ meson, with similar mass to that of the proton and rather long lifetime $\left(\tau_{\phi}=46.3 \pm 0.4 \mathrm{fm} / c\right.$ [50]) compared to that of the fireball, is an ideal candidate for such study. The yields of short-lived resonances such as the $\mathrm{K}^{* 0}\left(\tau_{\mathrm{K}^{* 0}}=\right.$ $4.16 \pm 0.05 \mathrm{fm} / c$ [50]) instead may be influenced by interactions during the hadronic phase: the re-scattering of the decay products in the fireball may prevent the detection of a fraction of the resonances, whereas pseudo-elastic hadron scattering can regenerate them. The effects of re-scattering and regeneration depend on the scattering cross section, the particle density, the particle lifetime and the timespan between chemical and kinetic freeze-out, namely the lifetime of the hadronic phase. Therefore, the observation of re-scattering effects would imply the presence of an extended hadronic phase. The latter can be studied by comparing particles with different lifetimes, such as the $\mathrm{K}^{* 0}$ resonance and the $\phi$ meson, which has a ten times longer lifetime. ALICE has observed [33] that in most central $\mathrm{Pb}-\mathrm{Pb}$ collisions at the LHC the $\mathrm{K}^{* 0} / \mathrm{K}$ ratio is significantly suppressed with respect to peripheral $\mathrm{Pb}-\mathrm{Pb}$ collisions, $\mathrm{pp}$ collisions and the value predicted by a statistical hadronisation model [51]. This is interpreted as a scenario where re-scattering during the hadronic phase, dominating for low-momentum resonances $\left(p_{\mathrm{T}}<2 \mathrm{GeV} / c\right)$ $[52,53]$, reduces the measurable yield of $\mathrm{K}^{* 0}$. No suppression is observed instead for the ten times longer-lived $\phi$, since it decays mainly after kinetic freeze-out. Based on these observations, a lower limit of $2 \mathrm{fm} / c$ on the lifetime of the hadronic phase in $0-20 \%$ most central $\mathrm{Pb}-\mathrm{Pb}$ events could be estimated [33]. The $\mathrm{K}^{* 0}$ suppression exhibits a monotonic trend with centrality, suggesting a dependence on the volume of the particle source at the kinetic freeze-out. A similar measurement of resonance production as a function of the system size in $\mathrm{p}-\mathrm{Pb}$ can provide information as regards the lifetime of the hadronic fireball produced in such a smaller system.

The $\mathrm{K}^{* 0}$ and $\phi$ mesons are reconstructed using the ALICE detector in $\mathrm{p}-\mathrm{Pb}$ collisions at $\sqrt{s_{\mathrm{NN}}}=5.02 \mathrm{TeV}$. Their yields, mean transverse momenta and ratios to identified long-lived hadrons in $\mathrm{p}-\mathrm{Pb}$ collisions are studied as a function of the system size or the multiplicity of the event, and compared with $\mathrm{pp}$ and $\mathrm{Pb}-\mathrm{Pb}$. The experimental conditions are briefly presented in Sect. 2. Section 3 illustrates the analysis procedure, including event and track selection, signal extraction, efficiency correction and systematic uncertainties. The results are presented in Sect. 4 and in Sect. 5 the conclusions are summarised.

\section{Experimental setup}

A complete description of the ALICE detector and its performance during the LHC Run I are reported in [54,55], respectively.

The analyses presented in this paper have been carried out on a sample of $\mathrm{p}-\mathrm{Pb}$ collision events at $\sqrt{s_{\mathrm{NN}}}=5.02 \mathrm{TeV}$ collected in 2013. The LHC configuration was such that the lead beam, with energy of $1.58 \mathrm{TeV}$ per nucleon, was circulating in the counter-clockwise direction, namely towards the ALICE "A" side (positive rapidity direction), while the $4 \mathrm{TeV}$ proton beam was circulating in the clockwise direction, towards the ALICE muon spectrometer, or " $C$ " side. According to this convention for the sign of the coordinates, the nucleon-nucleon center-of-mass system was moving in the laboratory frame with a rapidity of $y_{\mathrm{NN}}=-0.465$ in the direction of the proton beam. In the following, $y_{\text {lab }}\left(\eta_{\text {lab }}\right)$ are used to indicate the (pseudo) rapidity in the laboratory reference frame, whereas $y(\eta)$ denotes the (pseudo) rapidity in the nucleon-nucleon center-of-mass reference system.

For the results presented in this paper, a low-luminosity data sample has been analysed, consisting of events collected at an hadronic interaction rate of about $10 \mathrm{kHz}$. The interaction region had a root mean square of $6.3 \mathrm{~cm}$ along the beam direction and of about $60 \mu \mathrm{m}$ in the direction transverse to the beam. The event pile-up rate has been estimated to have negligible effects on the results of this analysis. In particular, pile-up of collisions from different bunch crossings is negligible due to the $200 \mathrm{~ns}$ bunch-crossing spacing, larger than the integration time of the zero-degree calorimeter (ZDC), while a small fraction of in-bunch pile-up events is removed by the offline analysis, as described in the next section.

Small acceptance forward detectors (V0, T0, and ZDC) are used for triggering, event characterisation, and multiplicity studies. The trigger is provided by two arrays of 32 scintillator detectors, VOA and VOC, that cover the full azimuthal angle in the pseudo-rapidity regions $2.8<\eta_{\text {lab }}<5.1(\mathrm{~Pb}-$ going direction) and $-3.7<\eta_{\text {lab }}<-1.7$ (p-going direction), respectively. V0 information is also used to classify events in multiplicity classes (see Sect. 2.1). The two quartz Cherenkov detectors T0A $\left(4.6<\eta_{\text {lab }}<4.9\right)$ and T0C $(-3.3$ $\left.<\eta_{\text {lab }}<-3\right)$ deliver the time and the longitudinal position of the interaction. The zero-degree calorimeters (ZDC), consisting of two tungsten-quartz neutron and two brass-quartz proton calorimeters placed symmetrically at a distance of 113 $\mathrm{m}$ from the interaction point, on both sides, are used to reject background and to count spectator nucleons.

The reconstruction of the primary vertex of the collision and the tracking of particles in the ALICE central barrel is provided by the inner tracking system (ITS) and the time-projection chamber (TPC), in the pseudo-rapidity range 
$\left|\eta_{\text {lab }}\right|<0.9$ and the full azimuthal angle. The ITS is a siliconbased detector, constituted by two innermost pixels layers (SPD), two intermediate drift (SDD) and two outer strip layers (SSD), with radii between 3.9 and $43 \mathrm{~cm}$ from the beam axis. The ALICE main tracker, the TPC, is a $90 \mathrm{~m}^{3}$ cylindrical drift chamber filled with $\mathrm{Ne}-\mathrm{CO}_{2}$ gas and divided in two parts by a central cathode. The end plates are equipped with multi-wire proportional chambers whose readout cathode pads allow one to sample particle tracks up to 159 points (clusters). In addition to tracking, the TPC allows particle identification via the specific ionisation energy loss $\mathrm{d} E / \mathrm{d} x$ in the gas.

The time-of-flight (TOF) detector, a large Multigap resistive plate chamber (MRPC) array covering $|\eta|<0.9$ and the full azimuthal angle, allows for particle identification at intermediate momenta and has been exploited together with the TPC for the analysis presented in this paper (see Sect. 3.1).

\subsection{Event selection}

The minimum bias trigger during $\mathrm{p}-\mathrm{Pb}$ data taking was configured to select hadronic events with high efficiency, by requiring a signal in either V0A or VOC. The resulting sample contains single-diffractive (SD), non-single-diffractive (NSD) and electromagnetic (EM) events. Diffractive interactions are described in Regge theory by the exchange of a colour singlet object with the quantum numbers of the vacuum (pomeron). In SD events one of the two nucleons breaks up producing particles in a limited rapidity interval. NSD events include double-diffractive interactions, where both nucleons break up by producing particles separated by a large rapidity gap, and other inelastic interactions. The offline analysis selects events having a coincidence of signals in both VOA and V0C in order to reduce the contamination from SD and EM events to a negligible amount. The trigger and eventselection efficiency for NSD events is estimated as $\epsilon_{\mathrm{NSD}}=$ 99.2\% using a combination of Monte-Carlo event generators, as described in $[48,49]$. The arrival time of signals on the $\mathrm{V0}$ and the $\mathrm{ZDC}$ is required to be compatible with a nominal $\mathrm{p}-\mathrm{Pb}$ collision occurring close to the nominal interaction point, to ensure the rejection of beam-gas and other machine-induced background collisions.

The primary vertex of the collision is determined using tracks reconstructed in the TPC and ITS. In case of low multiplicity events only the information from the SPD is used to reconstruct the vertex, as described in detail in [55]. $98.5 \%$ of all events have a primary vertex. Minimum bias events with the primary vertex positioned along the beam axis within $10 \mathrm{~cm}$ from the center of the ALICE detector are selected offline. A small fraction $(0.2 \%)$ of pile-up events from the same bunch crossing has been removed from the sample by rejecting events with multiple vertices. Events are accepted if the vertices separately measured by the SPD and using tracks are within $0.5 \mathrm{~cm}$, and if the SPD vertex is determined by at least five track segments defined by one hit in each one of the two layers of the detector.

After the trigger and offline event-selection criteria, the sample used for this analysis counts about $10^{8}$ events, corresponding to an integrated luminosity of about $50 \mu \mathrm{b}^{-1}$. The minimum bias sample has been further divided in several event classes based on the charged-particle multiplicity, estimated using the total charge deposited in the V0A detector positioned along the direction of the $\mathrm{Pb}$ beam. The yield of $\mathrm{K}^{* 0}$ is measured in five multiplicity classes, namely 0 $20,20-40,40-60,60-80$ and $80-100 \%$. In case of $\phi$ seven classes, namely $0-5,5-10,10-20,20-40,40-60,60-80$ and $80-100 \%$ are used. In addition, minimum bias spectra normalised to the fraction of NSD events are measured for both particles.

In order to study the dependence of particle production on the geometry of the collision, the V0A estimator for the charged particle multiplicity has been used to determine centrality, by following the approach based on the Glauber Monte Carlo model combined with a simple model for particle production [56,57], a strategy customarily employed in heavy-ion collisions [58]. The average number of binary collisions $\left\langle\mathrm{N}_{\text {coll }}\right\rangle$ (related to the number of participant nucleons $N_{\text {part }}$ by the simple relation $N_{\text {coll }}=N_{\text {part }}-1$ ), obtained with this method for each centrality class, are listed in Table 1 for future reference, together with the mean chargedparticle multiplicity density, $\left\langle\mathrm{d} N_{\mathrm{ch}} / \mathrm{d} \eta_{\mathrm{lab}}\right\rangle_{|\eta|<0.5}[47,48]$, here corrected for trigger and vertex-reconstruction inefficiency, which is about $5.5 \%$ in the lowest multiplicity event class. In addition, the average $N_{\text {coll }}$ has been determined with an hybrid method that uses the ZDC to classify the events

Table 1 Average charged-particle pseudo-rapidity density, $\left\langle\mathrm{d} N_{\mathrm{ch}} / \mathrm{d} \eta_{\mathrm{lab}}\right\rangle_{|\eta|<0.5}$, measured at mid-rapidity in visible cross section event classes and average number of colliding nucleons, $\left\langle\mathrm{N}_{\text {coll }}\right\rangle$. Multiplicity classes are defined using the V0A estimator [48,49], as described in the text. Total systematic uncertainties are reported, see [49] for details, which do not include the difference with respect to the other methods used to estimate the average $N_{\text {coll }}$. For minimum bias collisions, $\left\langle\mathrm{d} N_{\mathrm{ch}} / \mathrm{d} \eta_{\text {lab }}\right\rangle=16.81 \pm 0.71$ and $\left\langle\mathrm{N}_{\text {coll }}\right\rangle=6.87 \pm 0.5$

\begin{tabular}{lll}
\hline Multiplicity class $(\%)$ & $\left\langle\mathrm{d} N_{\text {ch }} / \mathrm{d} \eta_{\text {lab }}\right\rangle_{|\eta|<0.5}$ & $\left\langle\mathrm{~N}_{\text {coll }}\right\rangle$ \\
\hline $0-5$ & $45 \pm 1$ & $14.8 \pm 1.5$ \\
$5-10$ & $36.2 \pm 0.8$ & $13.0 \pm 1.3$ \\
$10-20$ & $30.5 \pm 0.7$ & $11.7 \pm 1.2$ \\
$0-20$ & $35.6 \pm 0.8$ & $12.8 \pm 1.3$ \\
$20-40$ & $23.2 \pm 0.5$ & $9.36 \pm 0.84$ \\
$40-60$ & $16.1 \pm 0.4$ & $6.42 \pm 0.46$ \\
$60-80$ & $9.8 \pm 0.2$ & $3.81 \pm 0.76$ \\
$80-100$ & $4.16 \pm 0.09$ & $1.94 \pm 0.45$ \\
\hline
\end{tabular}


according to the energy deposited by the neutrons emitted in the $\mathrm{Pb}$-going direction (by evaporation or fragmentation) or the energy measured with the ZDC in the $\mathrm{Pb}$-going direction and the assumption that the charged-particle multiplicity measured at mid-rapidity is proportional to the number of participant nucleons. This method was shown [49] to avoid possible bias in the event sample related to the fact that the range of multiplicities used to select a given class in $\mathrm{p}-\mathrm{Pb}$ collisions is of similar magnitude to the fluctuations on the same quantity. The variations of the average $N_{\text {coll }}$ for a given multiplicity class, obtained with different methods are found not to exceed $6 \%$ in any of the used classes.

\section{Resonance signal reconstruction}

$\mathrm{K}^{*}(892)^{0}$ and $\phi(1020)$ mesons are reconstructed through their decay into charged hadrons, $\mathrm{K}^{* 0} \rightarrow \mathrm{K}^{+} \pi^{-}$and $\overline{\mathrm{K}^{* 0}} \rightarrow \mathrm{K}^{-} \pi^{+}$, B.R. $=0.666$, and $\phi \rightarrow \mathrm{K}^{+} \mathrm{K}^{-}$, B.R. $=$ 0.489 [50]. Since $\mathrm{K}^{*}(892)^{0}$ and $\overline{\mathrm{K}^{*}}(892)^{0}$ are expected to be produced in equal amounts, as measured in lower energy experiments [59], for this measurement the yields of particle and anti-particle are combined in order to improve statistics. The average $\left(\mathrm{K}^{*}(892)^{0}+\overline{\mathrm{K}^{*}}(892)^{0}\right) / 2$ is indicated as $\mathrm{K}^{* 0}$ in the following. The $\phi(1020)$ meson is indicated as $\phi$.

For these measurements, the reconstructed $\mathrm{K}^{* 0}$ and $\phi$ are selected in the rapidity range $-0.5<y<0$, in order to ensure the best detector acceptance as the center of mass of the nucleon-nucleon system was moving with respect to the beam interaction point.

\subsection{Track selection and particle identification}

The charged tracks coming from the primary vertex of the collision ("primary" tracks) with $p_{\mathrm{T}}>0.15 \mathrm{GeV} / c$ and $\left|\eta_{\text {lab }}\right|$ $<0.8$ are considered for the invariant-mass reconstruction of $\mathrm{K}^{* 0}$ and $\phi$ in this analysis. The selection of primary tracks imposes the requirement that they satisfy good reconstruction quality criteria. It is required that tracks have left a signal in at least one of the layers of the SPD and that the distance of closest approach to the primary vertex of the collisions is lower than $7 \sigma_{x y}$ in the transverse plane and within $2 \mathrm{~cm}$ along the longitudinal direction. The resolution on the distance of closest approach in the transverse plane, $\sigma_{x y}$, is strongly $p_{\mathrm{T}^{-}}$ dependent and lower than $100 \mu \mathrm{m}$ for $p_{\mathrm{T}}>0.5 \mathrm{GeV} / c$ [55]. In addition tracks are required to cross at least 70 out of maximum 159 horizontal segments (or "rows") along the transverse readout plane of the TPC.

Primary tracks have been identified as $\pi$ or K based on the information of the TPC and TOF detectors. In the TPC, charged hadrons are identified by measuring the specific ionisation energy loss $(\mathrm{d} E / \mathrm{d} x)$ in the detector gas. With a resolution $\left(\sigma_{\mathrm{TPC}}\right)$ on $\mathrm{d} E / \mathrm{d} x$ of $6 \%$, the TPC allows a $2 \sigma_{\mathrm{TPC}}$ separation between $\pi$ and $\mathrm{K}$ up to $p_{\mathrm{T}} \sim 0.8 \mathrm{GeV} / c$ and above $3 \mathrm{GeV} / c$, in the relativistic rise region of the $\mathrm{d} E / \mathrm{d} x$. The TOF contributes to particle identification with the measurement of the time-of-flight of the particle, with the start time of the event measured by the $\mathrm{T} 0$ detector or using an algorithm which combines the particle arrival times at the TOF surface. In $\mathrm{p}-\mathrm{Pb}$ collisions, when the event time is determined by the TOF algorithm (available for $100 \%$ of the events which have more than three tracks) the resolution is $80<\sigma_{\mathrm{TOF}}<100$ ps. TOF allows a $2 \sigma_{\text {TOF }}$ separation between identified $\pi$ and $\mathrm{K}$ in the momentum range $0.7-3 \mathrm{GeV} / c$, and between $\mathrm{K}$ and protons up to $5 \mathrm{GeV} / c$ [60].

For the combined "TPC-TOF PID" approach, particles with a signal in the TOF are identified by requiring that the measured time-of-flight and energy loss do not deviate from the expected values for each given mass hypothesis by more than $2 \sigma_{\mathrm{TOF}}$ and $5 \sigma_{\mathrm{TPC}}$, respectively. For tracks which do not hit the TOF active region, a $2 \sigma_{\mathrm{TPC}}$ selection on the $\mathrm{d} E / \mathrm{d} x$ is applied. Variations of these cuts have been used for systematic studies, as described in Sect. 3.4. Besides the TPC-TOF, the measurement of $\phi$ has been performed following two alternative strategies, one which exploits a $2 \sigma_{T P C}$ separation on the particle energy loss in the TPC for the K identification, and the second for which no PID cuts are applied. In the noPID scheme all positively charged hadrons are considered as $\mathrm{K}^{+}$whereas all negatively charged hadrons are considered as $\mathrm{K}^{-}$. The no-PID approach extends the measurement of the yields from $p_{\mathrm{T}}=10 \mathrm{GeV} / c$, the upper limit reached by the PID analysis, to $16 \mathrm{GeV} / c$ (multiplicity dependent) or 21 $\mathrm{GeV} / c$ (minimum bias). At low $p_{\mathrm{T}}$, the TPC-TOF selection leads to a better separation between signal and background with respect to TPC-only and no-PID, therefore it is used until $p_{\mathrm{T}}(\phi)_{\text {cutoff }}=3 \mathrm{GeV} / c$. At high momentum, $\mathrm{K}$ and $\pi$ cannot be efficiently separated by TPC-TOF, therefore no-PID is used for $p_{\mathrm{T}}(\phi)>3 \mathrm{GeV} / c$ to maximise the total reconstruction efficiency. The multiplicity-integrated yields of $\phi$ (see Sect. 4) obtained with the no-PID, TPC only, and TPC-TOF approaches are compared in Fig. 1a in the common transverse momentum interval. Details of the signal extraction procedure and efficiency correction are given, respectively, in Sects. 3.2 and 3.3. The ratio of the data to the Lévy-Tsallis function (see Sect. 4.1) used to fit the TPC-TOF spectrum in the $0.3<p_{\mathrm{T}}<10 \mathrm{GeV} / c$ range (Fig. 1b) further shows good agreement among the three analyses, within uncertainties. In the case of $\mathrm{K}^{* 0}$, which is a wide resonance, PID is necessary also at high momentum to reduce the background and therefore the TPC-TOF strategy has been applied in the full kinematic range.

\subsection{Signal extraction}

$\mathrm{K}^{* 0}$ and $\phi$ signals are reconstructed in each multiplicity class and transverse momentum interval, as described in $[30,33]$. 


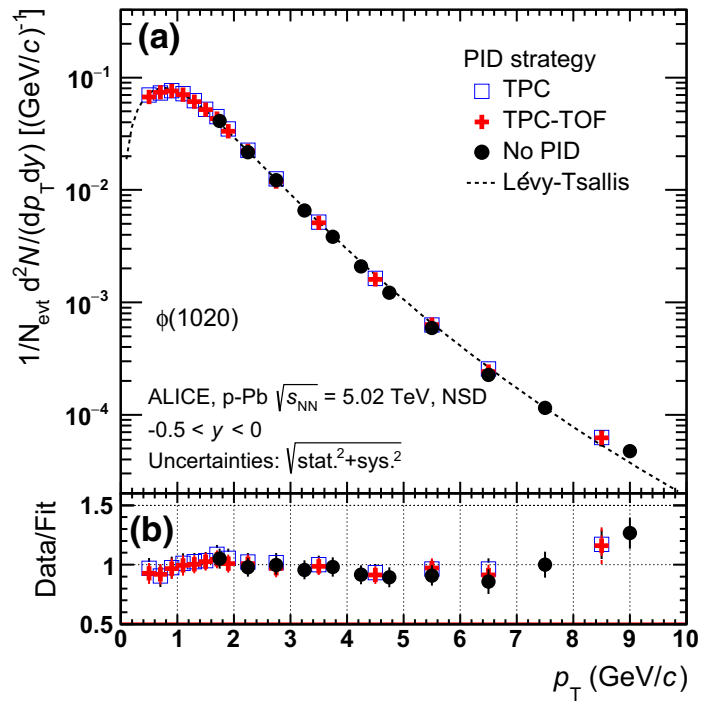

Fig. 1 a Comparison of the transverse momentum spectrum $\mathrm{d}^{2} N /\left(\mathrm{d} p_{\mathrm{T}} \mathrm{d} y\right)$ of $\phi$-meson in non-single-diffractive (NSD) $\mathrm{p}-\mathrm{Pb}$ events, reconstructed via the decay channel into $\mathrm{K}^{+} \mathrm{K}^{-}$by exploiting three different strategies for K identification: TPC only, TPC-TOF and no-PID. The reader can refer to Sect. 3.1 for details on the PID selection and to Sect. 3.2 for a description of the signal extraction procedure. The uncertainties are the sum in quadrature of statistical and systematic. A Lévy-Tsallis function (see Eq. 1) is used to fit the TPC-TOF spectrum in $0.3<p_{\mathrm{T}}<10 \mathrm{GeV} / c$. b Ratio of each spectrum to the fit function, showing good agreement of the three PID strategies within uncertainties

For each event, the invariant-mass distribution of the $\mathrm{K}^{* 0}(\phi)$ is constructed using all unlike-sign combinations of charged $\mathrm{K}$ candidates with $\pi(\mathrm{K})$ candidates. For $\mathrm{K}^{* 0}$ in the full momentum range and for $\phi$ up to $3 \mathrm{GeV} / c$ the TPC-TOF approach has been used for particle identification. $\phi$ mesons with $p_{\mathrm{T}}>3 \mathrm{GeV} / c$ have been reconstructed by applying no PID. In the following the $\mathrm{K}^{+}$and $\pi^{+}$candidates are labelled by $h^{+}$, the $\mathrm{K}^{-}$and $\pi^{-}$are labelled by $h^{-}$. The combinatorial background due to the uncorrelated pairs has been estimated in two ways, by the mixed-event technique and from the invariant-mass distribution of like-sign pairs from the same event. In the event-mixing method the shape of the uncorrelated background is estimated from the invariant-mass distribution of $h^{+} h^{-}$combinations from five different events. Effects from multiplicity fluctuations are minimised by dividing the sample into ten multiplicity classes and by performing event mixing within the same multiplicity class. In order to minimise distortions due to acceptance effects within each multiplicity class, the events are further sub-divided into 20 bins according to the relative vertex position along the $z$ axis $\left(\Delta z_{\mathrm{v}}=1 \mathrm{~cm}\right)$. The final mixed-event distribution for each multiplicity class is found by adding up the $M_{\text {inv }}$ distributions from each vertex $\Delta z_{v}$ interval. For the $\mathrm{K}^{* 0}$ analysis, the mixed-event distribution for each $p_{\mathrm{T}}$ bin is normalised by the smallest factor that leads to a positive-defined unlike-sign distribution after subtraction, within the statistical error in all invariant-mass bins. The mixed-event distribution for $\phi$ is normalised in the mass region $1.04<M_{\mathrm{KK}}<1.06 \mathrm{GeV} / c^{2}$. The normalisation range for $\mathrm{K}^{* 0}$ and $\phi$ is varied for systematic studies. In the like-sign technique, the invariant-mass distribution for the uncorrelated background is obtained by combining the $h^{+} h^{+}$and $h^{-} h^{-}$pairs from the same event according to a geometric mean $\left(2 \sqrt{\left(h^{+} h^{+}\right) \cdot\left(h^{-} h^{-}\right)}\right)$, in order to reduce statistical fluctuations in the resulting distribution. The like-sign background is subtracted without normalisation from the unlike-sign pairs distribution. The mixed-event method has been preferred for $\mathrm{K}^{* 0}(\phi)$ signal extraction in the range $0.4<p_{\mathrm{T}}<15 \mathrm{GeV} / c\left(0.3<p_{\mathrm{T}}<16 \mathrm{GeV} / c\right)$, given the smaller statistical uncertainties on the invariant-mass distribution. At very low momentum, $p_{\mathrm{T}}<0.4 \mathrm{GeV} / c$, the likesign distribution is found to reproduce better the background of the $\mathrm{K}^{* 0}$ and not to be affected by the choice of the normalisation range, therefore it has been preferred over the mixed event. Figure 2 shows the $M_{\mathrm{K} \pi}$ and $M_{\mathrm{KK}}$ invariantmass distributions before and after background subtraction in the transverse momentum interval $1.2 \leq p_{\mathrm{T}}<1.4 \mathrm{GeV} / c$, for the 0-20 and 0-5\% V0A multiplicity classes, for $\mathrm{K}^{* 0}$ and $\phi$, respectively.

After background subtraction, the resulting distributions exhibit a characteristic peak on top of a residual background (lower panels of Fig. 2). The latter is only partly due to imperfections in the description of the combinatorial background and mainly due to correlated pairs from jets, multi-body decay of heavier particles or correlated pairs contribution to the background from real resonance decays where the daughter particles are misidentified as K or $\pi$ by the TPC-TOF PID. A dedicated study in Monte Carlo simulations has been performed to ensure that the shape of the correlated background is a smooth function of mass and to verify that a second-order polynomial provides a good description of it.

As in [30], the signal peaks for $\mathrm{K}^{* 0}$ and $\phi$ are fitted, respectively, with a (non-relativistic) Breit-Wigner and a Voigtian function (convolution of Breit-Wigner and Gaussian) superimposed to a second-order polynomial function to shape the residual background. Examples are reported in the lower panels of Fig. 2, where fits are performed in the intervals 0.76 $<M_{\mathrm{K} \pi}<1.04 \mathrm{GeV} / c^{2}$ and $1.0<M_{\mathrm{KK}}<1.05 \mathrm{GeV} / c^{2}$. The fitting range is optimised for each $p_{\mathrm{T}}$ bin across all multiplicity event classes. The mass and width of $\mathrm{K}^{* 0}$ and $\phi$ are found to be compatible with the measurements in $\mathrm{Pb}-\mathrm{Pb}$ collisions [33]. For the measurement of the yields, the width of $\mathrm{K}^{* 0}$ and $\phi$ have been fixed to their natural values, $\Gamma\left(\mathrm{K}^{* 0}\right)=47.4 \pm$ $0.6 \mathrm{MeV} / c^{2}, \Gamma(\phi)=4.26 \pm 0.04 \mathrm{MeV} / c^{2}[50]$, whereas the resolution parameter of the Voigtian function for $\phi$ has been kept as a free parameter. The measured $p_{\mathrm{T}}$-dependent resolution on the $\phi$ mass (sigma of Gaussian) varies between 0.9 and $1.5 \mathrm{MeV} / c^{2}$, and it is consistent with the values extracted from Monte Carlo simulation. The sensitivity to the choice 


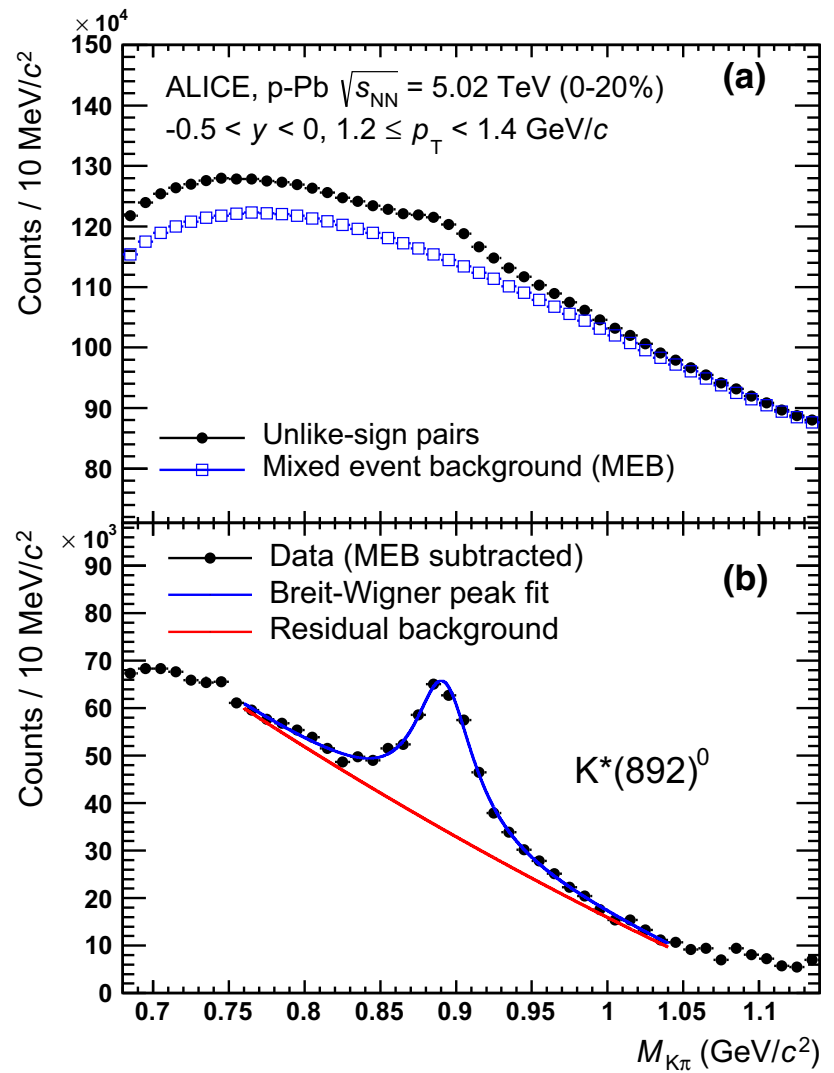

Fig. 2 Invariant-mass distributions for $\mathrm{K}^{* 0}(\mathbf{a}, \mathbf{b})$ and $\phi(\mathbf{c}, \mathbf{d})$ in the transverse momentum range $1.2 \leq p_{\mathrm{T}}<1.4 \mathrm{GeV} / c$ and multiplicity classes 0-20 and 0-5\%, respectively. Upper panels a, c, report the unlike-sign invariant-mass distribution and the mixed-event background (MEB) normalised as described in the text. In lower panels $\mathbf{b}, \mathbf{c}$,

of the normalisation interval, the fitting range, the shape of the background function, the fitting range and the constraints on mass, width and resolution parameters has been studied by varying the default settings, as described in Sect. 3.4.

In minimum bias collisions the sample of reconstructed particles includes about $3.4 \times 10^{6} \mathrm{~K}^{* 0}$ and $8.6 \times 10^{5} \phi$ in the transverse momentum range $0<p_{\mathrm{T}}\left(\mathrm{K}^{* 0}\right)<15 \mathrm{GeV} / c$ and $0.3<p_{\mathrm{T}}(\phi)<21 \mathrm{GeV} / c$, respectively. With the available statistics, the $\mathrm{K}^{* 0}$ production in the $80-100 \%$ V0A multiplicity event class has been measured up to $p_{\mathrm{T}}=6 \mathrm{GeV} / c$, while the $\phi$ spectra extend up to $16 \mathrm{GeV} / c$ in the $0-60 \%$ multiplicity percentile interval and up to $13 \mathrm{GeV} / c$ in $60-80$ and $80-100 \%$.

\subsection{Detector acceptance and efficiency}

In order to evaluate the detector acceptance and reconstruction efficiency, a sample of about $10^{8}$ Monte Carlo simulated $\mathrm{p}-\mathrm{Pb}$ events, based on the DPMJET 3.05 event generator

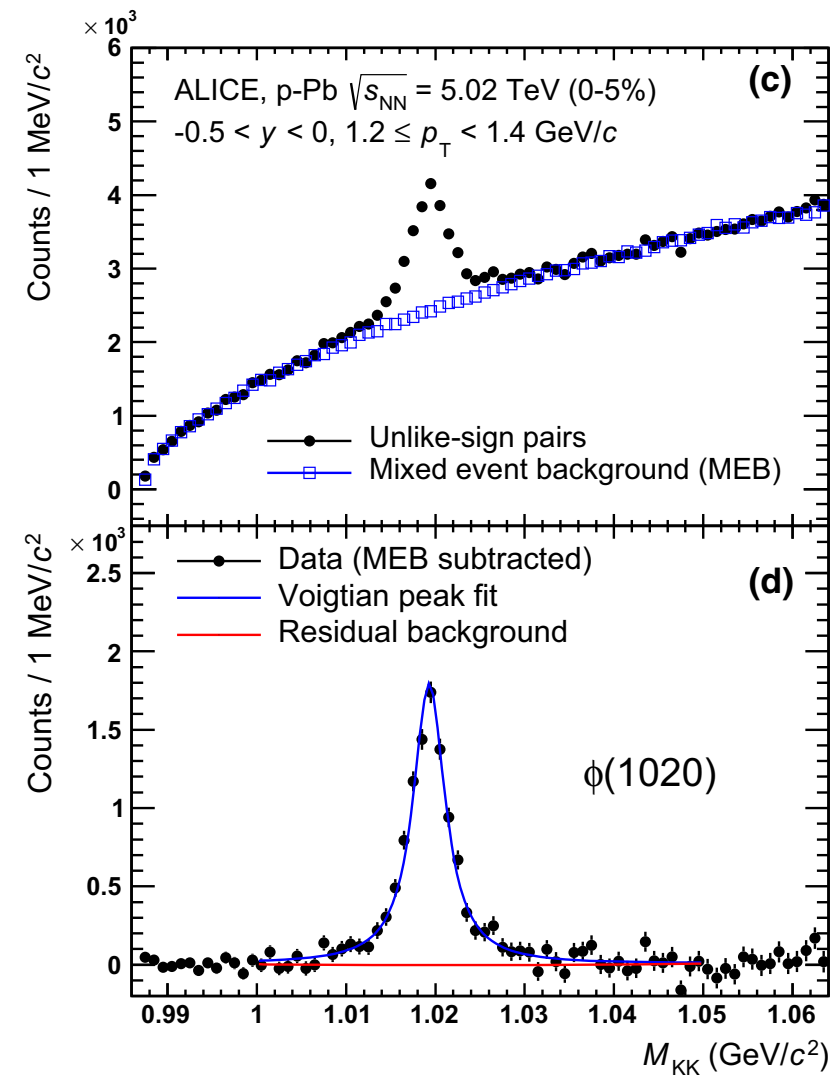

the distributions after background subtraction are shown. The $\mathrm{K}^{* 0}$ peak is fitted with a Breit-Wigner function whereas the $\phi$ meson peak is described by a Voigtian function. A second-order polynomial function is used to describe the residual background

[61], with the detector geometry and material budget modelled by GEANT 3.21 [62], has been analysed. The acceptance and efficiency correction is determined as the fraction of generated resonances in the rapidity interval $-0.5<y<0$ that have been reconstructed. The reconstructed signal pairs are obtained upon combination of primary $\pi$ and $\mathrm{K}$ selected by applying the same kinematics cuts and track cuts as in the data (see Sect. 3.1), including TPC-TOF PID cuts for $\mathrm{K}^{* 0}$, and $\phi$ with $p_{\mathrm{T}}<3 \mathrm{GeV} / c$. For $\phi$ with $p_{\mathrm{T}}>3 \mathrm{GeV} / c$ no PID cuts are applied. The acceptance and efficiency corrections, Acc $\times \epsilon$, for $\mathrm{K}^{* 0}$ and $\phi$ are reported in Fig. 3 as a function of $p_{\mathrm{T}}$ for minimum bias events. Since only events with reconstructed primary vertex have been considered in the computation of $(\operatorname{Acc} \times \epsilon)\left(p_{\mathrm{T}}\right)$, a correction factor has to be applied to the total number of accepted events in each V0A multiplicity event class, to account for vertex reconstruction inefficiency. The correction is about 0.995 for $60-80 \%$ class and 0.945 for the lowest multiplicity events $80-100 \%$, and it is applied as discussed in Sect. 4.1. 


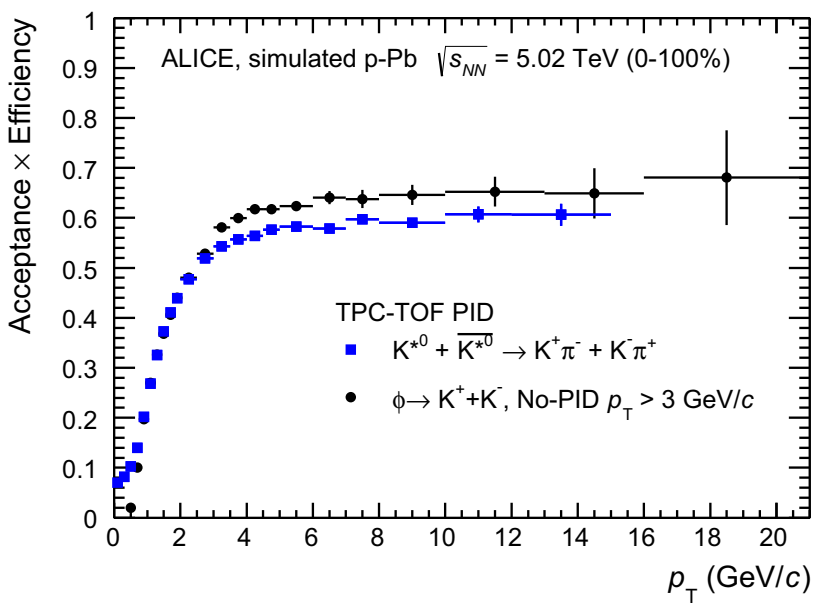

Fig. 3 Detector acceptance and signal reconstruction efficiency for $\mathrm{K}^{* 0}$ and $\phi$ mesons, which includes reconstruction, track selection and particle identification efficiency. For $\mathrm{K}^{* 0}$ and $\phi$ production below $p_{\mathrm{T}}<3 \mathrm{GeV} / c$, the PID efficiency is relative to the TPC-TOF approach, whereas for $\phi$ production with $p_{\mathrm{T}}>3 \mathrm{GeV} / c$ no PID contribution is included, as no particle identification is applied in the analysis

\subsection{Systematic uncertainties}

The measurement of $\mathrm{K}^{* 0}$ and $\phi$ production in $\mathrm{p}-\mathrm{Pb}$ collisions have been tested for systematic effects due to global tracking efficiency, track selection cuts, PID, signal extraction, knowledge of the material budget and of the hadronic interaction cross section in the detector material, as summarised in Table 2. The approach is similar to the one adopted for the study of $\mathrm{K}^{* 0}$ and $\phi$ in $\mathrm{Pb}-\mathrm{Pb}$ collisions [33], but the total average uncertainty evaluated in the $\mathrm{p}-\mathrm{Pb}$ case is significantly lower (about half of the relative uncertainty in the $\mathrm{Pb}-\mathrm{Pb}$ ), mainly due to lower contributions from global tracking effi- ciency and the signal extraction procedure. No multiplicity dependence of systematic effects has been observed, therefore the uncertainties presented in Sect. 2 have been averaged among all multiplicity event classes. For each particle, they are quoted for two separate momentum intervals: for $\mathrm{K}^{* 0}$, one can distinguish a low- $p_{\mathrm{T}}$ range $\left(0<p_{\mathrm{T}}\left(\mathrm{K}^{* 0}\right)<4 \mathrm{GeV} / c\right)$ where the knowledge of the material budget and hadronic interaction cross section in the detector material enter in the systematic uncertainty, as opposite to the high- $p_{\mathrm{T}}$ range (4 $\left.<p_{\mathrm{T}}\left(\mathrm{K}^{* 0}\right)<15 \mathrm{GeV} / c\right)$ where these contributions are negligible $(<0.5 \%)$. In the $\phi$ case, two $p_{\mathrm{T}}$ intervals are considered, according to the particle identification approach used to identify the decay products, namely the "TPC-TOF" and "No PID" strategies described in Sect. 3.1. The $p_{\mathrm{T}}$ region where the TPC-TOF PID is applied $\left(p_{\mathrm{T}}<3 \mathrm{GeV} / c\right)$, coincides also with the range where effects of material budget and hadronic interaction cross section are relevant for the measurement of $\phi$ production.

The main source of uncertainty, common to $\mathrm{K}^{* 0}$ and $\phi$, comes from the determination of the global tracking efficiency. In $\mathrm{p}-\mathrm{Pb}$ collisions this contribution has been estimated to be a $p_{\mathrm{T}}$-independent effect of $3 \%$ for charged particles [48], which results in a $6 \%$ effect when any two tracks are combined in the invariant-mass analysis of $\mathrm{K}^{* 0}$ and $\phi$.

The track selection was varied to study systematic effects: the analyses are sensitive to variations of the cuts on the number of crossed rows in the TPC and the distance of closest approach to the primary vertex of the collision. Track selection enters in the total uncertainty with a relative contribution of $2.5 \%$ for $\mathrm{K}^{* 0}$ and about $1.9-2.2 \%$ for the $\phi$ case.

At high transverse momentum, namely for $p_{\mathrm{T}}\left(\mathrm{K}^{* 0}\right)>8$ $\mathrm{GeV} / c$ and $p_{\mathrm{T}}(\phi)>12 \mathrm{GeV} / c$, the systematic uncertainties are dominated by the raw yield extraction procedure. This
Table 2 Sources of systematic uncertainties for $\mathrm{K}^{* 0}$ and $\phi$ yields $\left(\mathrm{d}^{2} N /\left(\mathrm{d} p_{\mathrm{T}} \mathrm{d} y\right)\right)$. For each source and transverse momentum range (see text for details), the average relative uncertainty over all multiplicity classes is listed. For each $p_{\mathrm{T}}$ range, the particle identification ("PID technique") used for the analysis is also indicated. The contributions have been summed in quadrature to estimate the total relative systematic uncertainty

\begin{tabular}{|c|c|c|c|c|}
\hline \multirow{3}{*}{$\begin{array}{l}p_{\mathrm{T}}(\mathrm{GeV} / c) \\
\text { PID technique }\end{array}$} & \multicolumn{2}{|l|}{$\mathrm{K}^{* 0}$} & \multicolumn{2}{|l|}{$\phi$} \\
\hline & $0-4.0$ & $4.0-15.0$ & $0.3-3.0$ & $3.0-21.0$ \\
\hline & \multicolumn{2}{|c|}{ TPC-TOF } & TPC-TOF & No PID \\
\hline Global tracking efficiency & \multicolumn{2}{|c|}{$6 \%$} & \multicolumn{2}{|c|}{$6 \%$} \\
\hline Track selection cuts & \multicolumn{2}{|c|}{$2.5 \%$} & $1.9 \%$ & $2.2 \%$ \\
\hline Material budget & $1.2 \%$ & $<0.5 \%$ & $2.2 \%$ & $<0.5 \%$ \\
\hline Hadronic interaction cross section & $1.9 \%$ & $<0.5 \%$ & $2.4 \%$ & $<1 \%$ \\
\hline Particle identification & $1.1 \%$ & $2.7 \%$ & $0.9 \%$ & - \\
\hline Signal extraction & $3.8 \%$ & $4.6 \%$ & $1.8 \%$ & $4.3 \%$ \\
\hline Total & $7.9 \%$ & $8.4 \%$ & $7.4 \%$ & $7.7 \%$ \\
\hline
\end{tabular}


contribution is labelled as "Signal extraction" in Table 2 and it includes the background normalisation region, the choice of the fitting range, the residual background shape and variations of the constraints on the fit parameters. In addition to the default strategy described in Sect. 3.2, the mixedevent background distributions for $\mathrm{K}^{* 0}$ and $\phi$ have been normalised in different invariant-mass regions that surround, but exclude the signal peaks. The sensitivity of the $\mathrm{K}^{* 0}(\phi)$ yield extraction to the fit range has been studied by varying each interval boundary within $\pm 50 \mathrm{MeV} / c^{2}\left( \pm 5 \mathrm{MeV} / c^{2}\right)$. As alternative to the second-order polynomial, third- and first-order polynomial functions have been used to fit the residual background. The measurements for both $\mathrm{K}^{* 0}$ and $\phi$ turned out to be independent on the mass parameters, but not on the constraints on the $\mathrm{K}^{* 0}$ width and $\phi$ mass resolution. Therefore, the $\mathrm{K}^{* 0}$ width has been varied by $\pm 50 \%$ for systematic studies, while the $\phi$ resolution has been varied within the range of values observed in the simulation. Due to the lower particle multiplicity and the improved PID strategy that has led to a lower residual background after mixed-event background subtraction, the contribution of signal extraction for $\mathrm{K}^{* 0}$ is reduced by half in $\mathrm{p}-\mathrm{Pb}$ with respect to the $\mathrm{Pb}-\mathrm{Pb}$ case, where the uncertainty associated to the choice of the fitting range was larger than $9 \%$ [33].

In order to study the effect of the PID selection on signal extraction, the cuts on TOF and TPC have been varied to $3 \sigma$ and $4 \sigma$ with respect to the default settings described in Sect. 3.1, resulting in the average contribution to the systematic uncertainty reported in Table 2 as "particle identification". For $\mathrm{K}^{* 0}$ the average contribution from PID is $1.1 \%$ in the low- $p_{\mathrm{T}}$ range, and $2.7 \%$ at high transverse momenta. The contribution to the $\phi$ uncertainty is $0.9 \%$ on average in the transverse momentum range where TPC-TOF PID is applied.

The knowledge of the material budget contributes for $\mathrm{K}^{* 0}(\phi)$ with an average of $1.2 \%(2.2 \%)$ at low transverse momentum, and a maximum of $3.5 \%(5.4 \%)$, reached for 0 $<p_{\mathrm{T}}<0.2 \mathrm{GeV} / c\left(0.8<p_{\mathrm{T}}<0.9 \mathrm{GeV} / c\right)$. In both cases, it is negligible for $p_{\mathrm{T}}>3 \mathrm{GeV} / c$. The contribution from the estimate of the hadronic interaction cross section in the detector material is $1.9 \%(2.4 \%)$ for $\mathrm{K}^{* 0}(\phi)$ at low $p_{\mathrm{T}}$, negligible for $p_{\mathrm{T}}>4 \mathrm{GeV} / c\left(p_{\mathrm{T}}>3 \mathrm{GeV} / c\right)$. These effects were evaluated by combining the uncertainties for a $\pi$ and a $\mathrm{K}$ (for $\mathrm{K}^{* 0}$ ), and for two $\mathrm{K}$ (in the case of $\phi$ ), determined as in $[42,47]$, according to the kinematics of the decay.

The systematics were studied independently for all event classes, in order to separate the sources which are multiplicitydependent and uncorrelated across multiplicity bins. In particular, signal extraction and PID are fully uncorrelated sources, whereas global tracking, track cuts, material budget and hadronic cross section are correlated among different event classes.

\section{Results and discussion}

\subsection{Transverse momentum spectra}

The multiplicity-dependent transverse momentum spectra of $\mathrm{K}^{* 0}$ and $\phi$ mesons measured in the rapidity range $-0.5<y$ $<0$ are reported in Fig. 4. Measured yields are corrected for acceptance, efficiency and branching ratio, and normalised to the visible cross section in each V0A multiplicity event class, as discussed in Sect. 3.3. The minimum bias spectra for $\mathrm{K}^{* 0}$ and $\phi$ are also reported in Fig. 4 and have been
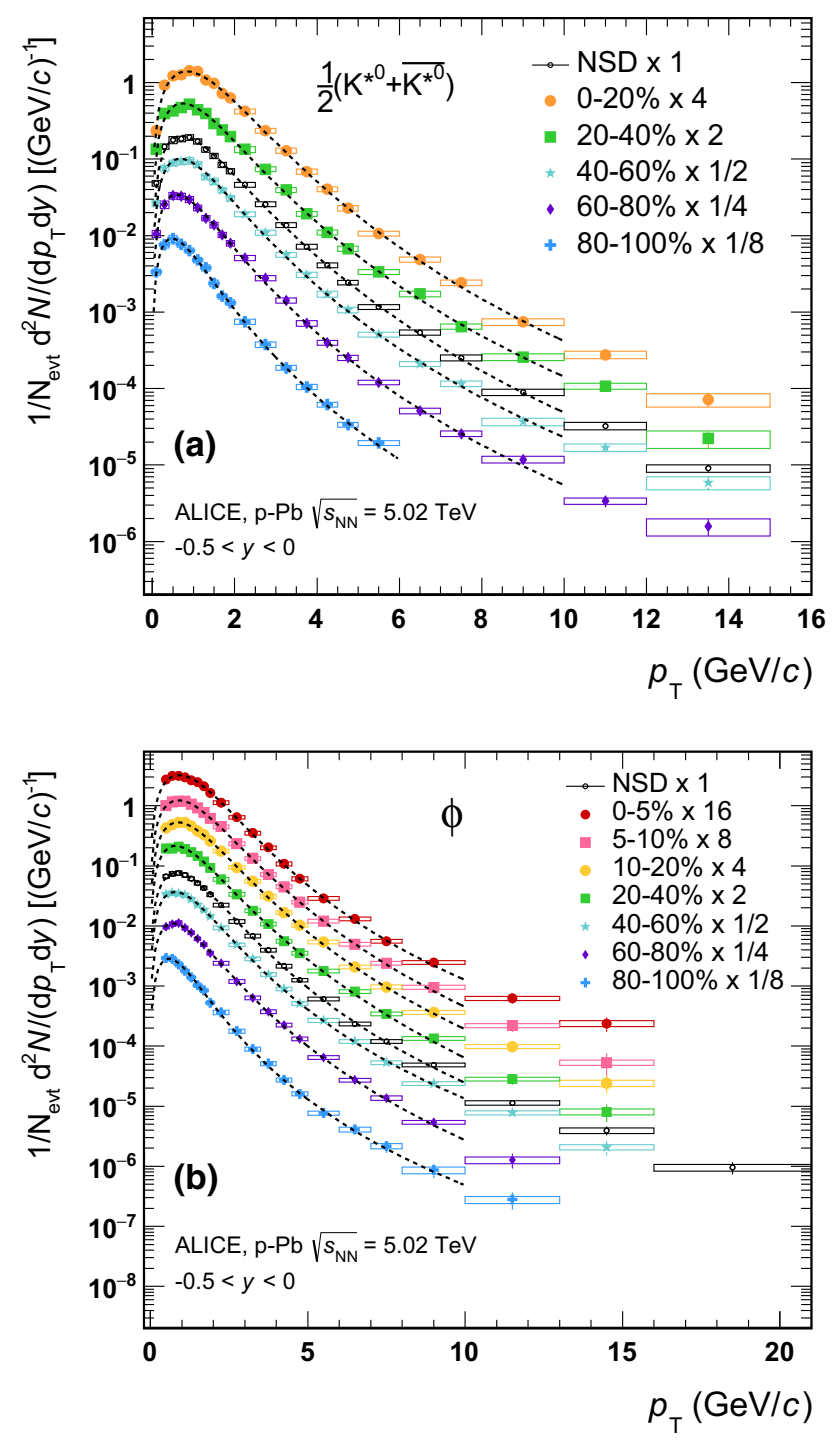

Fig. 4 Transverse momentum spectra $\mathrm{d}^{2} N /\left(\mathrm{d} p_{\mathrm{T}} \mathrm{d} y\right)$ of $\mathrm{K}^{* 0}$ (a) and $\phi$ (b) for different multiplicity classes (V0A estimator), measured in the rapidity range $-0.5<y<0 . \mathrm{K}^{* 0}$ and $\overline{\mathrm{K}^{* 0}}$ are averaged. The multiplicity-dependent spectra are normalised to the visible cross section, whereas the minimum bias spectrum is normalised to the fraction of NSD events (see text). Statistical (bars) and systematic (boxes) uncertainties are indicated. Dashed lines represent Lévy-Tsallis fits; see text for details 
Table 3 Parameters of the Lévy-Tsallis fit function and values of $\phi$ and $\mathrm{K}^{* 0} \mathrm{~d} N / \mathrm{d} y$ and $\left\langle p_{\mathrm{T}}\right\rangle$ for different multiplicity classes. The $C$ and $n$ parameters with their statistical uncertainty, the reduced $\chi^{2}$ of the fit and the fraction of the total yield obtained by extrapolation ("Extr.") are reported. The yields and $\left\langle p_{\mathrm{T}}\right\rangle$ are obtained considering data in the measured range and using the result of the fit in the extrapolation region, and are listed as (value \pm stat. \pm uncorr. \pm corr.), where the errors are the statistical uncertainty, the uncorrelated and correlated contributions to the systematic uncertainty, respectively. In the $\left\langle p_{T}\right\rangle$ case, the contribution to the systematic uncertainty correlated across multiplicity classes is negligible. The minimum bias spectrum has been normalised to the fraction of non-single-diffractive events (NSD) and an additional $3.1 \%$ relative contribution from the normalisation to NSD has to be considered in the systematic uncertainty on $\mathrm{d} N / \mathrm{d} y$

\begin{tabular}{lllllll}
\hline Multiplicity $(\%)$ & $C(\mathrm{GeV})$ & $n$ & $\chi^{2} / \mathrm{ndf}$ & Extr. & $\mathrm{d} N / \mathrm{d} y$ (data + extr. $)$ & $\left\langle p_{\mathrm{T}}\right\rangle(\mathrm{GeV} / c)$ \\
\hline $\mathrm{K}^{* 0}$ & & & & & & \\
$0-20$ & $0.440 \pm 0.010$ & $11.1 \pm 0.5$ & 1.7 & $<10^{-4}$ & $0.616 \pm 0.008 \pm 0.037 \pm 0.037$ & $1.379 \pm 0.011 \pm 0.020$ \\
$20-40$ & $0.430 \pm 0.009$ & $9.7 \pm 0.4$ & 1.7 & $<10^{-4}$ & $0.426 \pm 0.006 \pm 0.026 \pm 0.026$ & $1.300 \pm 0.010 \pm 0.019$ \\
$40-60$ & $0.359 \pm 0.008$ & $8.8 \pm 0.3$ & 0.5 & $<10^{-4}$ & $0.302 \pm 0.004 \pm 0.019 \pm 0.018$ & $1.211 \pm 0.009 \pm 0.017$ \\
$60-80$ & $0.309 \pm 0.008$ & $7.8 \pm 0.3$ & 0.6 & $<10^{-4}$ & $0.185 \pm 0.003 \pm 0.013 \pm 0.011$ & $1.108 \pm 0.009 \pm 0.021$ \\
$80-100$ & $0.224 \pm 0.008$ & $6.2 \pm 0.3$ & 0.4 & 0.002 & $0.083 \pm 0.001 \pm 0.005 \pm 0.005$ & $0.943 \pm 0.009 \pm 0.016$ \\
NSD & $0.388 \pm 0.003$ & $9.4 \pm 0.1$ & 1.8 & $<10^{-4}$ & $0.315 \pm 0.002 \pm 0.018 \pm 0.018$ & $1.270 \pm 0.005 \pm 0.017$ \\
$\phi$ & & & & & & \\
$0-5$ & $0.472 \pm 0.010$ & $12.5 \pm 0.9$ & 1.5 & 0.094 & $0.377 \pm 0.004 \pm 0.020 \pm 0.023$ & $1.437 \pm 0.009 \pm 0.028$ \\
$5-10$ & $0.469 \pm 0.010$ & $12.0 \pm 0.8$ & 1.1 & 0.094 & $0.288 \pm 0.003 \pm 0.014 \pm 0.017$ & $1.442 \pm 0.009 \pm 0.025$ \\
$10-20$ & $0.453 \pm 0.010$ & $11.3 \pm 0.6$ & 1.2 & 0.097 & $0.244 \pm 0.002 \pm 0.012 \pm 0.014$ & $1.421 \pm 0.008 \pm 0.024$ \\
20-40 & $0.413 \pm 0.009$ & $9.8 \pm 0.4$ & 1.1 & 0.105 & $0.185 \pm 0.001 \pm 0.009 \pm 0.011$ & $1.357 \pm 0.006 \pm 0.025$ \\
$40-60$ & $0.382 \pm 0.009$ & $8.8 \pm 0.4$ & 0.6 & 0.115 & $0.1229 \pm 0.0008 \pm 0.0064 \pm 0.0073$ & $1.310 \pm 0.006 \pm 0.031$ \\
$60-80$ & $0.349 \pm 0.009$ & $8.3 \pm 0.4$ & 0.5 & 0.115 & $0.0695 \pm 0.0006 \pm 0.0037 \pm 0.0041$ & $1.242 \pm 0.008 \pm 0.024$ \\
$80-100$ & $0.260 \pm 0.010$ & $6.7 \pm 0.3$ & 0.4 & 0.163 & $0.0297 \pm 0.0004 \pm 0.0023 \pm 0.0018$ & $1.055 \pm 0.010 \pm 0.030$ \\
NSD & $0.412 \pm 0.014$ & $10.0 \pm 0.5$ & 0.8 & 0.106 & $0.1344 \pm 0.0005 \pm 0.0069 \pm 0.0081$ & $1.355 \pm 0.003 \pm 0.030$ \\
\hline
\end{tabular}

normalised to the number of NSD events after applying the correction for trigger efficiency and event selection $\left(\epsilon_{N S D}\right)$, vertex reconstruction $\left(\epsilon_{v t x}\right)$ and vertex selection described in Sect. 2 , resulting in a total scaling factor of 0.964 .

The $p_{\mathrm{T}}$-integrated particle yields, $\mathrm{d} N / \mathrm{d} y$, and mean transverse momentum, $\left\langle p_{\mathrm{T}}\right\rangle$, are determined by using the transverse momentum spectra in the measured range and by using a fit function to extrapolate the yield in the $p_{\mathrm{T}}$ range where no measurement is available. The same procedure is applied to the spectra of $\mathrm{K}^{* 0}$ and $\phi$ for each event class. The LévyTsallis parameterisation [63] has been chosen to fit the corrected $\mathrm{d}^{2} N /\left(\mathrm{d} p_{\mathrm{T}} \mathrm{d} y\right)$ spectra, as it has successfully been adopted to fit the particle spectra in pp collisions at RHIC and at LHC [30,64-66]. The Lévy-Tsallis functional form describes the shape of the exponential spectra at low transverse momentum and the power law distributions at large $p_{\mathrm{T}}$ with an inverse slope parameter $C$ and an exponent parameter $n$

$$
\begin{aligned}
\frac{\mathrm{d}^{2} N}{\mathrm{~d} p_{T} \mathrm{~d} y}= & p_{T} \frac{\mathrm{d} N}{\mathrm{~d} y} \frac{(n-1)(n-2)}{n C\left[n C+m_{0}(n-2)\right]} \\
& \times\left[1+\frac{\sqrt{p_{T}^{2}+m_{0}^{2}}-m_{0}}{n C}\right]^{-n},
\end{aligned}
$$

where $m_{0}$ is the mass of the particle, $n, C$ and the integrated yields $\mathrm{d} N / \mathrm{d} y$ are the free parameters. The fits are performed in the $p_{\mathrm{T}}$ range where the Lévy-Tsallis function provides a satisfactory description of each spectrum, namely in the interval $0-10 \mathrm{GeV} / c$ for $\mathrm{K}^{* 0}$ and $0.3-10.0 \mathrm{GeV} / c$ for $\phi$. The values of the fit parameters $C$ and $n$, as well as the reduced $\chi^{2}$ are reported in Table 3 , together with the $\mathrm{d} N / \mathrm{d} y$ and $\left\langle p_{\mathrm{T}}\right\rangle$ obtained using the data and the fit function in the extrapolation region.

For $\mathrm{K}^{* 0}$ the extrapolation, necessary only at high $p_{\mathrm{T}}$, covers a fraction of the total yield lower than $0.1 \%$. For $\phi$ the extrapolated yield is dominated by the fraction of signal in the low transverse momentum region, which constitutes about $10.6 \%$ of the total in the minimum bias case. For all multiplicity classes this fraction is reported in Table 3 . It can be noticed that the inverse slope parameter $C$ and the exponent parameter $n$ increase with multiplicity, reflecting the flattening of the spectra from peripheral to most central events.

The uncertainty on $\mathrm{d} N / \mathrm{d} y$ and $\left\langle p_{\mathrm{T}}\right\rangle$ is dominated by systematics, which include the contribution of the $p_{\mathrm{T}^{-}}$ uncorrelated systematic uncertainty on the measured spectrum (in average about $6.3 \%$ for $\mathrm{K}^{* 0}, 3.6 \%$ for $\phi$ ), the $p_{\mathrm{T}}$-correlated contributions from global tracking efficiency ( $6 \%$ for $\mathrm{K}^{* 0}$ and $\phi$, only on $\mathrm{d} N / \mathrm{d} y$ ), and the extrapolation of the yield. The first contribution has been estimated by repeating the Lévy-Tsallis fits moving the measured points within their systematic uncertainties, whereas in order to evaluate the latter, a blast-wave function [67] has been used alterna- 
tively to fit the spectra. The relative systematic uncertainty on $\mathrm{d} N / \mathrm{d} y$ due to the choice of the fit function varies between 1.5 and $3 \%$ for $\phi$, going from high to low multiplicity. Such a contribution is negligible in the case of $\mathrm{K}^{* 0}$, due to the fact that its production is measured down to zero transverse momentum.

\subsection{Mean transverse momentum}

In a hydrodynamically evolving system the spectral shapes are driven by the expansion velocity, thus by the mass of the particle and they are expected to follow "mass ordering". Vice versa, the observation of mass ordering of particle spectra may be suggestive of the presence of collective (hydrodynamic) behaviour of the system. Although the presence of a strong radial flow is established in $\mathrm{Pb}-\mathrm{Pb}$ collisions [42], the measurements in $\mathrm{p}-\mathrm{Pb}$ are not conclusive [47], as the comparison between data and models for pp collisions that incorporate final-state effects (such as colour reconnection), shows that the latter could mimic the presence of radial flow. The measurements of $\mathrm{K}^{* 0}$ and $\phi$ can further probe the presence of "mass ordering", since they have a similar mass to the proton.

The transverse momentum spectra of $\mathrm{K}^{* 0}$ and $\phi$, reported in Fig. 4, become flatter, thus harder, going from the most peripheral to the most central $\mathrm{p}-\mathrm{Pb}$ events. In other words, the mean transverse momentum increases with multiplicity. This is also shown in Fig. 5, where the $\left\langle p_{\mathrm{T}}\right\rangle$ of $\mathrm{K}^{* 0}$ and $\phi$ as a function of the average charged-particle multiplicity density $\left(\left\langle\mathrm{d} N_{\mathrm{ch}} / \mathrm{d} \eta_{\mathrm{lab}}\right\rangle_{|\eta|<0.5}\right)$ is compared to that of other identified hadrons, including $\pi^{ \pm}, \mathrm{K}^{ \pm}, \mathrm{K}_{S}^{0}, \mathrm{p}, \Lambda, \Xi^{-}$and $\Omega^{-}$, in $\mathrm{p}-\mathrm{Pb}$ collisions at $\sqrt{s_{\mathrm{NN}}}=5.02 \mathrm{TeV}$ [47,68]. Going from the lowest to the highest multiplicity events, the relative increase of $\left\langle p_{\mathrm{T}}\right\rangle$ for $\mathrm{K}^{* 0}$ and $\phi$ mesons is about $40 \%$, common to a wide variety of particles, including $\mathrm{K}^{ \pm}, \mathrm{K}_{S}^{0}, \Lambda, \Xi^{ \pm}$ and $\Omega^{ \pm}$. The relative increase is smaller for $\pi$ (about $26 \%$ ) but larger for protons (about $52 \%$ ). The $\left\langle p_{\mathrm{T}}\right\rangle$ of $\mathrm{K}^{* 0}$ is about $10 \%$ larger than that of proton in all event classes and compatible with $\left\langle p_{\mathrm{T}}\right\rangle$ of $\Lambda$. The $\left\langle p_{\mathrm{T}}\right\rangle$ of $\phi$ is instead about $20 \%$ larger than proton and between $4 \%(0-5 \%)$ and $8 \%(80-$ $100 \%$ ) larger than $\Lambda$. A similar hierarchy is also observed in pp collisions and peripheral $\mathrm{Pb}-\mathrm{Pb}$ collisions, but not in central $\mathrm{Pb}-\mathrm{Pb}$ collisions, where, as expected from hydrodynamics, particles with similar mass have similar $\left\langle p_{\mathrm{T}}\right\rangle$.

In Fig. 6 the $\left\langle p_{\mathrm{T}}\right\rangle$ of $\mathrm{K}^{* 0}$, proton and $\phi$ are compared in the three collision systems as a function of the cubic root of the average charged-particle multiplicity density, $\left\langle\mathrm{d} N_{\mathrm{ch}} / \mathrm{d} \eta_{\text {lab }}\right\rangle^{1 / 3}$. Based on the observation that the femtoscopic radii scale approximately linearly with $\left\langle\mathrm{d} N_{\mathrm{ch}} / \mathrm{d} \eta_{\mathrm{lab}}\right\rangle^{1 / 3}[20]$, this observable is used as a proxy for the system size, associated with the radius of the fireball at freeze-out. In $\mathrm{p}-\mathrm{Pb}$, where no extended hadronic medium is expected to be formed, the system size can be associated to

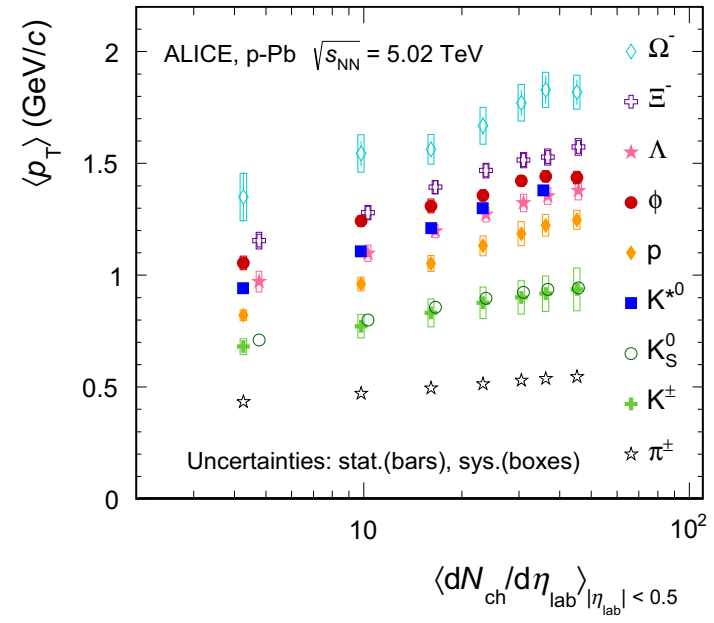

Fig. 5 Mean transverse momentum of $\mathrm{K}^{* 0}$ and $\phi$ compared to that of identified $\pi^{ \pm}, \mathrm{K}^{ \pm}, \mathrm{K}_{S}^{0}$, p, $\Lambda, \Xi^{-}$and $\Omega^{-}$previously measured by ALICE in $\mathrm{p}-\mathrm{Pb}$ collisions at $\sqrt{s_{\mathrm{NN}}}=5.02 \mathrm{TeV}[47,68]$ as a function of the charged-particle density measured in the pseudo-rapidity range $\left|\eta_{\text {lab }}\right|<0.5\left(\left\langle\mathrm{~d} N_{\mathrm{ch}} / \mathrm{d} \eta_{\text {lab }}\right\rangle_{|\eta|<0.5}\right)$. The $\mathrm{K}_{S}^{0}, \Lambda$ and $\Xi^{-}$points are slightly displaced along the $x$-axis to avoid superposition with other points. Statistical uncertainties are represented as bars, whereas boxes indicate systematic uncertainties

the width of the distribution of the particle emission points. The argument holding for $\mathrm{Pb}-\mathrm{Pb}$ has been extended in this paper also to the proton-nucleus case, based on the linear trend of the measured radii with $\left\langle\mathrm{d} N_{\mathrm{ch}} / \mathrm{d} \eta_{\mathrm{lab}}\right\rangle^{1 / 3}$ in $\mathrm{p}-\mathrm{Pb}$ collisions [69]. From Fig. 6 one can see that at similar event multiplicity, the $\left\langle p_{\mathrm{T}}\right\rangle$ is larger in $\mathrm{p}-\mathrm{Pb}$ than in $\mathrm{Pb}-\mathrm{Pb}$ and the increase with multiplicity is steeper. An analogous observation for unidentified charged particles has been reported in [70], where it is shown that in models of pp collisions, the strong increase in $\left\langle p_{\mathrm{T}}\right\rangle$ with $\left\langle N_{\mathrm{ch}}\right\rangle$ can be understood as the effect of colour reconnection between strings produced in multi-parton interactions. Considering that for a given multiplicity class in $\mathrm{p}-\mathrm{Pb}$ and peripheral $\mathrm{Pb}-\mathrm{Pb}$ events the geometry of the collision and the dynamics of the systems are different but the production of $\mathrm{K}^{* 0}$ and $\phi$ mesons relative to long-lived hadrons is comparable (see Sect. 4.4), one can conclude that the sample of $\mathrm{p}-\mathrm{Pb}$ collisions is dominated by events with a larger fraction of quadri-momentum transferred, thus "harder".

In central $\mathrm{Pb}-\mathrm{Pb}$ collisions, $\left\langle p_{\mathrm{T}}\right\rangle$ of $\mathrm{K}^{* 0}$, proton and $\phi$ are consistent within uncertainties (Fig. 6) and follow "mass ordering". This is consistent with the hypothesis that particle boost in the hadronic phase is driven by radial flow $[33,42]$. This mass ordering seems to weaken going towards peripheral $\mathrm{Pb}-\mathrm{Pb}$ collisions, where it is only approximate. In $\mathrm{p}-\mathrm{Pb}$ and minimum bias $\mathrm{pp}$ collisions $\left\langle p_{\mathrm{T}}\right\rangle(\phi)>\left\langle p_{\mathrm{T}}\right\rangle\left(\mathrm{K}^{* 0}\right)>\left\langle p_{\mathrm{T}}\right\rangle(p)$.

The $\left\langle p_{\mathrm{T}}\right\rangle$ for several particles as a function of their mass for $0-20 \% \mathrm{p}-\mathrm{Pb}$ at $\sqrt{s_{\mathrm{NN}}}=5.02 \mathrm{TeV}$ and minimum bias pp collisions at $\sqrt{s}=7 \mathrm{TeV}[30,64,66]$ are illustrated in 


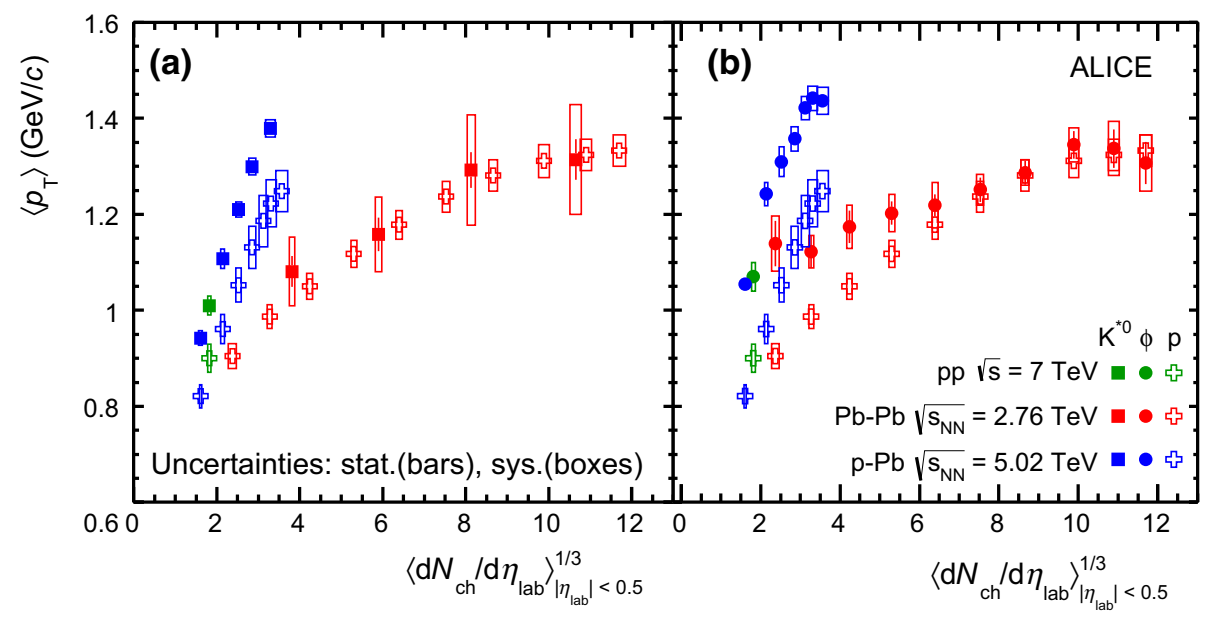

Fig. 6 System size dependence of the mean transverse momentum of $\mathrm{K}^{* 0}$ compared to that of the proton (left panel) and $\left\langle p_{\mathrm{T}}\right\rangle$ of $\phi$ compared to that of the proton (right panel). The system size is defined as the cubic root of the average charged particle multiplicity density $\left(\left\langle\mathrm{d} N_{\mathrm{ch}} / \mathrm{d} \eta_{\text {lab }}\right\rangle^{1 / 3}\right)$ measured in the ALICE central barrel $\left(\left|\eta_{\text {lab }}\right|<0.5\right)$

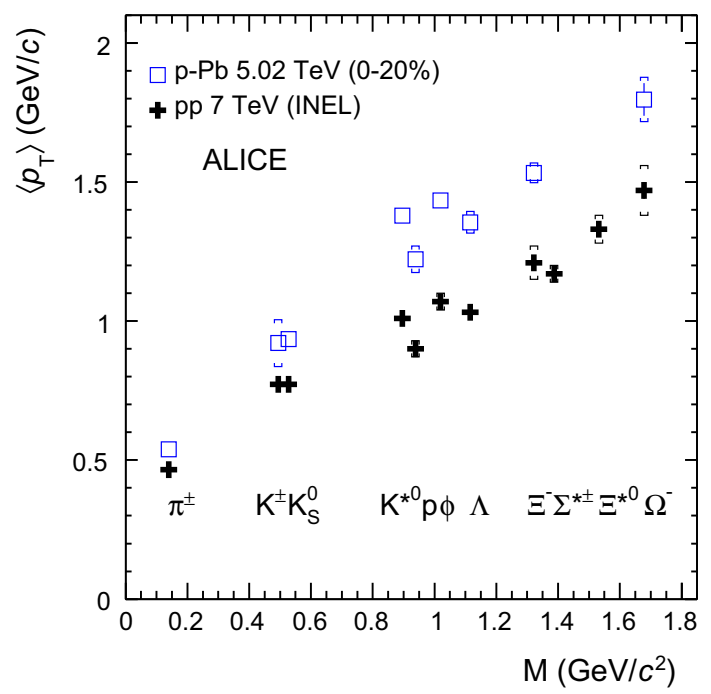

Fig. 7 Mass dependence of the mean transverse momentum of identified particles measured by ALICE in minimum bias pp collisions at $\sqrt{s}=7 \mathrm{TeV}[30,64,66]$ and $0-20 \% \mathrm{p}-\mathrm{Pb}$ collisions $[47,68]$. Statistical uncertainties are represented as bars, boxes indicate total systematic uncertainties

Fig. 7. In $\mathrm{p}-\mathrm{Pb}$, the $\left\langle p_{\mathrm{T}}\right\rangle$ of all particles but $\mathrm{K}^{* 0}$ has been obtained as the average between the available measured values weighted by the particle integrated yields in $0-5,5-10$ and $10-20 \%[47,68]$. For $\mathrm{K}^{* 0}$, the direct measurement of $\left\langle p_{\mathrm{T}}\right\rangle$ in $0-20 \%$ is available (see Table 3 ). For the pp case, also the recent measurements on the short-lived baryonic resonances $\Sigma(1385)^{ \pm}$and $\Xi(1530)^{0}$ (indicated as $\Sigma^{* \pm}$ and $\Xi^{* 0}$ ) have been included in the comparison. The mean trans- in pp at $\sqrt{s}=7 \mathrm{TeV}$ (green) [30,64], $\mathrm{p}-\mathrm{Pb}$ at $\sqrt{s_{\mathrm{NN}}}=5.02 \mathrm{TeV}$ (blue) $[47,68]$ and $\mathrm{Pb}-\mathrm{Pb}$ at $\sqrt{s_{\mathrm{NN}}}=2.76 \mathrm{TeV}$ (red) [33,42]. Statistical uncertainties are represented as bars, boxes indicate total systematic uncertainties

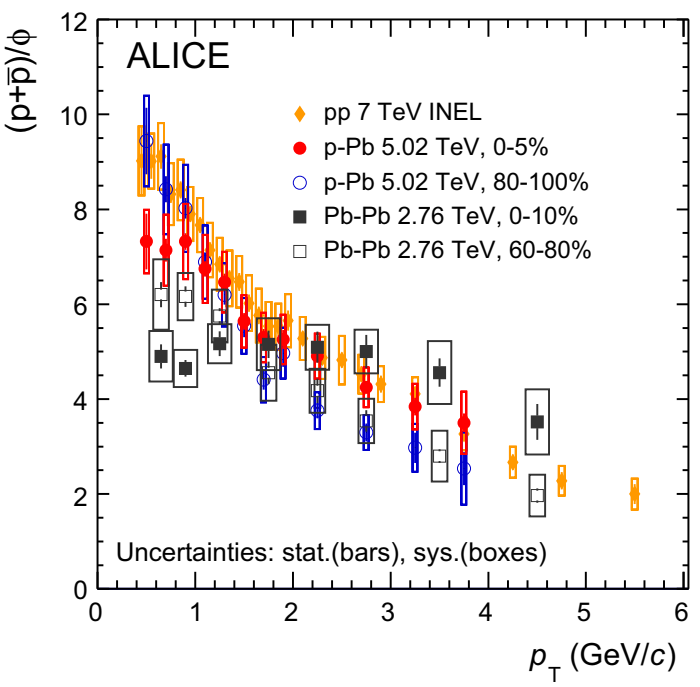

Fig. 8 The $(\mathrm{p}+\overline{\mathrm{p}}) / \phi$ ratio measured in $\mathrm{p}-\mathrm{Pb}$ in $0-5$ and $80-100 \%$ V0A multiplicity classes, compared to the same ratio measured in minimum bias pp collision at $\sqrt{s}=7 \mathrm{TeV}[30,64], 0-10 \%$ central and 60-80\% peripheral $\mathrm{Pb}-\mathrm{Pb}$ collisions at $\sqrt{s_{\mathrm{NN}}}=2.76 \mathrm{TeV}$ [33]

verse momentum is larger for larger masses, but Fig. 7 shows that in $\mathrm{pp}$ and $\mathrm{p}-\mathrm{Pb}$ collisions the $\left\langle p_{\mathrm{T}}\right\rangle$ values for $\mathrm{K}^{* 0}$ and $\phi$ mesons are systematically larger with respect to a linear trend which includes protons and $\Lambda$ instead. These results seem to suggest that a different type of scaling holds in $\mathrm{pp}$ and $\mathrm{p}-\mathrm{Pb}$ collisions and prepare the way for a more detailed investigation, which is, however, outside of the scope of this paper. 


\subsection{Differential $(\mathrm{p}+\overline{\mathrm{p}}) / \phi$ ratio}

The multiplicity dependence of the $(\mathrm{p}+\overline{\mathrm{p}}) / \phi$ ratio as a function of transverse momentum is studied to compare the spectral shapes of $\phi$ mesons and protons [47]. The differential ratios for the 0-5 and 80-100\% V0A multiplicity event classes in $\mathrm{p}-\mathrm{Pb}$ collisions are reported in Fig. 8 together with the ratios in minimum bias pp collisions, $0-10 \%$ central and $60-80 \%$ peripheral $\mathrm{Pb}-\mathrm{Pb}$. In peripheral $\mathrm{p}-\mathrm{Pb}$ the $(\mathrm{p}+\overline{\mathrm{p}}) / \phi$ ratio exhibits a qualitatively similar steep decrease as in pp collisions, and it is consistent with the ratio measured in 80-90\% peripheral $\mathrm{Pb}-\mathrm{Pb}$ collisions ([33], not shown in Fig. 8). The flat behaviour of $(\mathrm{p}+\overline{\mathrm{p}}) / \phi$ for $p_{\mathrm{T}}<3 \mathrm{GeV} / c$ in $0-10 \%$ central $\mathrm{Pb}-\mathrm{Pb}$ collisions has been previously discussed in [33] and found to be consistent with the expectations of hydrodynamic models. In the $0-5 \% \mathrm{p}-\mathrm{Pb}$, a hint of flattening is observed for $p_{\mathrm{T}}<1.5 \mathrm{GeV} / c$, but systematic uncertainties are such that no conclusive evidence can be derived. Despite being about 10-20\% larger but compatible within uncertainties, the best qualitative agreement of $(\mathrm{p}+\overline{\mathrm{p}}) / \phi$ in highmultiplicity $\mathrm{p}-\mathrm{Pb}(0-5 \%$ V0A multiplicity event class $)$ is achieved with respect to the $60-80 \%$ peripheral $\mathrm{Pb}-\mathrm{Pb}$ collisions, which has also a similar particle multiplicity.

\subsection{Integrated particle ratios}

Particle ratios are useful observables to study particle production mechanisms by comparing particles with similar or different strangeness content, mass and lifetime. Short-lived particles such as $\mathrm{K}^{* 0}$ and $\phi$ are used in heavy-ion collisions to derive information on the lifetime of the hadronic phase and on the mechanisms which take place before the kinetic freeze-out, such as re-scattering and regeneration. If dominant over regeneration, re-scattering is expected to reduce the observed yield of resonances, especially at low momentum and in high particle density environments [52]. For the $\mathrm{K}^{* 0}$ resonance re-scattering is the dominant effect at play in most central $\mathrm{Pb}-\mathrm{Pb}$ collisions (and at low transverse momentum, $p_{\mathrm{T}}<2 \mathrm{GeV} / c$ ). This observation comes from the strong centrality dependence of the $\mathrm{K}^{* 0} / \mathrm{K}$ ratio (see Fig. 9 ) and its direct comparison with the ratio of the longer-lived $\phi$ meson relative to $\mathrm{K}$ [33].

For $\mathrm{p}-\mathrm{Pb}$ collisions, the ratios of $\mathrm{K}^{* 0}$ and $\phi$-meson production to that of long-lived hadrons have been computed starting from the integrated yields of $\pi, \mathrm{K}$ and proton measured by ALICE with the same data sample [47], and they are reported for each multiplicity class in Table 4.

The systematic uncertainty on tracking, track selection, material budget and hadronic interaction cross section are correlated among each particle and its decay products, thus they partially cancel out in the propagation of the error to the final ratio. The residual uncertainties after cancellation are correlated across the event classes. Systematic uncertainties

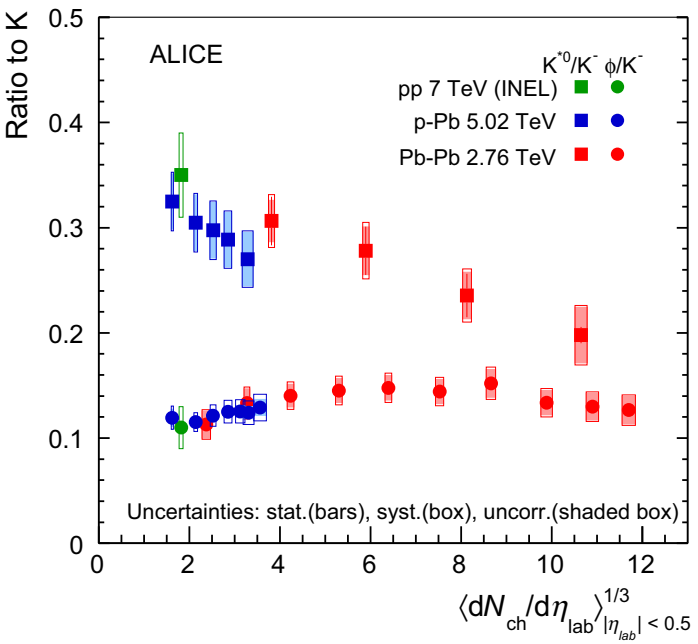

Fig. 9 Ratio of $\mathrm{K}^{* 0}$ and $\phi$ to charged $\mathrm{K}$ measured in the three collision systems, as a function of the cube root of the average charged-particle density $\left(\left\langle\mathrm{d} N_{\mathrm{ch}} / \mathrm{d} \eta_{\mathrm{lab}}\right\rangle^{1 / 3}\right)$ measured at mid-rapidity, used as a proxy for the system size. Squares represent $\mathrm{K}^{* 0} / \mathrm{K}$, circles refer to $\phi / \mathrm{K}$. Statistical uncertainties (bars) are shown together with total (hollow boxes) and multiplicity-uncorrelated (shaded boxes) systematic uncertainties. Measurements in pp at $\sqrt{s}=7 \mathrm{TeV}$ and $\mathrm{Pb}-\mathrm{Pb}$ collisions at $\sqrt{s_{\mathrm{NN}}}=2.76 \mathrm{TeV}$ are taken from [30] and [33], respectively

derived from signal extraction and PID selection are uncorrelated.

Based on the results reported in Table 4, one can conclude that no significant multiplicity dependence is observed in the $\mathrm{K}^{* 0} / \pi$ and the $\mathrm{K}^{* 0} / \mathrm{p}$ ratios. The $2 \phi /\left(\pi^{+}+\pi^{-}\right)$ratio exhibits instead an increasing trend with multiplicity, going from $0.0143 \pm 0.001$ in the lowest multiplicity bin to 0.0185 \pm 0.001 in the highest multiplicity class, for a total increase of $29 \%$ with a $2.6 \sigma$ significance. A similar trend with multiplicity is also observed for the $2 \phi /(\mathrm{p}+\overline{\mathrm{p}})$ ratio, which increases by about $24 \%$ with a significance of $1.3 \sigma$ going from $80-100$ to $0-5 \%$.

The increase of the $2 \phi /\left(\pi^{+}+\pi^{-}\right)$ratio with multiplicity can be interpreted in the context of strangeness enhancement. The enhancement of $\phi$-meson ( $\mathrm{s} \overline{\mathrm{s}})$ production relative to pion has been observed in $\mathrm{Pb}-\mathrm{Pb}$ to follow the enhancement observed for other strange and multi-strange baryons [33]. In $\mathrm{p}-\mathrm{Pb}$ the results are in general agreement with the results on $\Xi / \pi$ and $\Omega / \pi$ ratio [68], which seem to indicate that the strangeness content may control the rate of increase with multiplicity.

Most interesting are the ratios of $\mathrm{K}^{* 0}$ and $\phi$ to charged $\mathrm{K}$, which have been compared to similar measurements in $\mathrm{pp}$ at $\sqrt{s}=7 \mathrm{TeV}[30]$ and $\mathrm{Pb}-\mathrm{Pb}$ collisions at $\sqrt{s_{\mathrm{NN}}}=$ $2.76 \mathrm{TeV}$ [33], looking for indications of the presence of re-scattering effects in central $\mathrm{p}-\mathrm{Pb}$ collisions. $\mathrm{K}^{* 0} / \mathrm{K}$ and $\phi / \mathrm{K}$ in the three collision systems are reported in Fig. 9 as a function of $\left\langle\mathrm{d} N_{\mathrm{ch}} / \mathrm{d} \eta_{\mathrm{lab}}\right\rangle^{1 / 3}$. While spanning a smaller range of particle multiplicities, the $\mathrm{K}^{* 0} / \mathrm{K}$ and $\phi / \mathrm{K}$ ratios in 
Table 4 Ratio of $\mathrm{K}^{* 0}$ resonance and $\phi$-meson yields to long-lived hadrons [47], for different multiplicity classes in $\mathrm{p}-\mathrm{Pb}$ collisions at $\sqrt{s_{\mathrm{NN}}}=5.02 \mathrm{TeV}$. The results are reported as value \pm stat. \pm sys. (uncorr.), where the first error is the statistical uncertainty, the second is the total systematic uncertainty and the value in parentheses indicates the component of uncertainty uncorrelated across multiplicity classes

\begin{tabular}{llll}
\hline Multiplicity (\%) & $\left(\mathrm{K}^{* 0}+\overline{\mathrm{K}^{* 0}}\right) /\left(\pi^{+}+\pi^{-}\right)$ & $\left(\mathrm{K}^{* 0}+\overline{\mathrm{K}^{* 0}}\right) /\left(\mathrm{K}^{+}+\mathrm{K}^{-}\right)$ & $\left(\mathrm{K}^{* 0}+\overline{\mathrm{K}^{* 0}}\right) /(\mathrm{p}+\overline{\mathrm{p}})$ \\
\hline $\mathrm{K}^{* 0}$ & & & $0.676 \pm 0.009 \pm 0.062(0.059)$ \\
$0-20$ & $0.0379 \pm 0.0006 \pm 0.0028(0.0026)$ & $0.270 \pm 0.004 \pm 0.027(0.026)$ & $0.698 \pm 0.009 \pm 0.063(0.060)$ \\
20-40 & $0.0392 \pm 0.0006 \pm 0.0029(0.0027)$ & $0.289 \pm 0.004 \pm 0.027(0.026)$ & $0.700 \pm 0.009 \pm 0.064(0.060)$ \\
$40-60$ & $0.0395 \pm 0.0006 \pm 0.0030(0.0028)$ & $0.298 \pm 0.004 \pm 0.028(0.026)$ & $0.696 \pm 0.009 \pm 0.065(0.061)$ \\
$60-80$ & $0.0393 \pm 0.0006 \pm 0.0032(0.0029)$ & $0.308 \pm 0.004 \pm 0.028(0.026)$ & $0.745 \pm 0.011 \pm 0.067(0.063)$ \\
$80-100$ & $0.0399 \pm 0.0006 \pm 0.0030(0.0028)$ & $0.325 \pm 0.005 \pm 0.028(0.026)$ & $2 \phi /(\mathrm{p}+\overline{\mathrm{p}})$ \\
\hline Multiplicity (\%) & $2 \phi /\left(\pi^{+}+\pi^{-}\right)$ & $2 \phi /\left(\mathrm{K}^{+}+\mathrm{K}^{-}\right)$ & \\
\hline$\phi$ & & & $0.331 \pm 0.003 \pm 0.030(0.016)$ \\
$0-5$ & $0.0185 \pm 0.0002 \pm 0.0014(0.0009)$ & $0.1290 \pm 0.0013 \pm 0.0126(0.0076)$ & $0.311 \pm 0.003 \pm 0.028(0.012)$ \\
$5-10$ & $0.0174 \pm 0.0002 \pm 0.0013(0.0006)$ & $0.1241 \pm 0.0013 \pm 0.0112(0.0057)$ & $0.310 \pm 0.003 \pm 0.027(0.011)$ \\
$10-20$ & $0.0174 \pm 0.0001 \pm 0.0012(0.0006)$ & $0.1254 \pm 0.0010 \pm 0.0110(0.0053)$ & $0.303 \pm 0.002 \pm 0.026(0.011)$ \\
$20-40$ & $0.0170 \pm 0.0001 \pm 0.0012(0.0006)$ & $0.1250 \pm 0.0008 \pm 0.0107(0.0053)$ & $0.286 \pm 0.002 \pm 0.025(0.011)$ \\
$40-60$ & $0.0161 \pm 0.0001 \pm 0.0012(0.0006)$ & $0.1213 \pm 0.0009 \pm 0.0102(0.0052)$ & $0.261 \pm 0.003 \pm 0.022(0.010)$ \\
$60-80$ & $0.0147 \pm 0.0001 \pm 0.0011(0.0006)$ & $0.1143 \pm 0.0012 \pm 0.0089(0.0046)$ & $0.267 \pm 0.004 \pm 0.028(0.018)$ \\
$80-100$ & $0.0143 \pm 0.0002 \pm 0.0013(0.0009)$ & $0.1160 \pm 0.0018 \pm 0.0110(0.0078)$ &
\end{tabular}

$\mathrm{p}-\mathrm{Pb}$ cover within uncertainties the range of values measured in peripheral (40-60 and 60-80\%) $\mathrm{Pb}-\mathrm{Pb}$ and $\mathrm{pp}$ collisions.

In order to quantify the evolution of the $\mathrm{p}-\mathrm{Pb}$ ratios with multiplicity class, the ratios $(y)$ have been fitted with a firstorder polynomial, $y=a x+b$, where $x=\left\langle\mathrm{d} N_{\mathrm{ch}} / \mathrm{d} \eta_{\mathrm{lab}}\right\rangle^{1 / 3}$. Only the statistical and uncorrelated systematic uncertainties, added in quadrature, have been considered for the purpose of the fit. In $\mathrm{p}-\mathrm{Pb}$ collisions the $\phi / \mathrm{K}$ ratio follows the trend from minimum bias pp to peripheral $\mathrm{Pb}-\mathrm{Pb}$ collisions. The linear fit to the $\mathrm{p}-\mathrm{Pb}$ data returns a positive but small slope parameter, $\mathrm{a}_{\phi}=0.008 \pm 0.004$. A similar fit to the $\mathrm{K}^{* 0} / \mathrm{K}$ ratio in $\mathrm{p}-\mathrm{Pb}$ instead results in a negative slope, $\mathrm{a}_{\mathrm{K} * 0}=-0.030 \pm 0.018$. The pp value for the $\mathrm{K}^{* 0} / \mathrm{K}$ ratio is consistent with the ratio in the lowest multiplicity $\mathrm{p}-\mathrm{Pb}$ events. The slope obtained fitting the $\mathrm{Pb}-\mathrm{Pb}$ data, $\mathrm{a}^{\prime}{ }_{\mathrm{K}}^{* 0}=-0.016 \pm 0.006$, is interpreted as due to re-scattering effects in central collisions [33]. The slopes in $\mathrm{Pb}-\mathrm{Pb}$ and $\mathrm{p}-\mathrm{Pb}$ are compatible within the uncertainties (about $60 \%$ in $\mathrm{p}-\mathrm{Pb}$ and $27 \%$ for $\mathrm{Pb}-\mathrm{Pb}$ ), and the decreasing trend in $\mathrm{K}^{* 0} / \mathrm{K}$ may be a hint of the presence of re-scattering effects in highmultiplicity $\mathrm{p}-\mathrm{Pb}$ events and indicative for a finite lifetime of the hadronic phase in $\mathrm{p}-\mathrm{Pb}$ collisions. Further comparisons with models of $\mathrm{p}-\mathrm{Pb}$ collisions which include resonances and re-scattering effects would be useful to distinguish between the different scenarios.

\section{Conclusions}

The production of $\mathrm{K}^{* 0}$ resonances and $\phi$ mesons in $\mathrm{p}-\mathrm{Pb}$ collisions at $\sqrt{s_{\mathrm{NN}}}=5.02 \mathrm{TeV}$ has been measured with the ALICE detector, including multiplicity-dependent transverse momentum spectra, mean transverse momentum and particle ratios to long-lived light-flavoured hadron production. The system size dependence of these observables has been studied by comparing the $\mathrm{p}-\mathrm{Pb}$ results with previous measurements in $\mathrm{Pb}-\mathrm{Pb}$ and $\mathrm{pp}$ collisions. In all collision systems, the mean transverse momentum increases with multiplicity for all particle species. The mass ordering observed in central $\mathrm{Pb}-\mathrm{Pb}$ collisions, where particle with similar mass have similar $\left\langle p_{\mathrm{T}}\right\rangle$, can be attributed to the presence of radial flow. In $\mathrm{p}-\mathrm{Pb}$ as well as in pp collisions $\left\langle p_{\mathrm{T}}\right\rangle$ mass ordering is not observed. The measurement of $\left\langle p_{\mathrm{T}}\right\rangle$ for other hadronic species could shed more light on whether the observed effect is due to the mesonic (baryonic) nature of the particles, or instead, this behaviour is common to resonances rather than long-lived hadrons. Ratios of $\mathrm{K}^{* 0}$ and $\phi$ production to charged $\mathrm{K}$ are found to be in agreement with the ratios measured at similar multiplicities in $\mathrm{pp}$ and $\mathrm{Pb}-\mathrm{Pb}$ collisions. The measurements in $\mathrm{p}-\mathrm{Pb}$ follow the trend observed in $\mathrm{Pb}-\mathrm{Pb}$ within the accessible multiplicity range and the uncertainties. The $\mathrm{K}^{* 0} / \mathrm{K}$ ratio exhibits a finite negative slope from the lowest to the highest multiplicity $\mathrm{p}-\mathrm{Pb}$ events, sugges- 
tive of a finite lifetime of the hadronic phase in the small $\mathrm{p}-\mathrm{Pb}$ system.

Acknowledgments The ALICE Collaboration would like to thank all its engineers and technicians for their invaluable contributions to the construction of the experiment and the CERN accelerator teams for the outstanding performance of the LHC complex. The ALICE Collaboration gratefully acknowledges the resources and support provided by all Grid centres and the Worldwide LHC Computing Grid (WLCG) Collaboration. The ALICE Collaboration acknowledges the following funding agencies for their support in building and running the ALICE detector: State Committee of Science, World Federation of Scientists (WFS) and Swiss Fonds Kidagan, Armenia; Conselho Nacional de Desenvolvimento Científico e Tecnológico (CNPq), Financiadora de Estudos e Projetos (FINEP), Fundação de Amparo à Pesquisa do Estado de São Paulo (FAPESP); National Natural Science Foundation of China (NSFC), the Chinese Ministry of Education (CMOE) and the Ministry of Science and Technology of China (MSTC); Ministry of Education and Youth of the Czech Republic; Danish Natural Science Research Council, the Carlsberg Foundation and the Danish National Research Foundation; The European Research Council under the European Community's Seventh Framework Programme; Helsinki Institute of Physics and the Academy of Finland; French CNRS-IN2P3, the 'Region Pays de Loire', 'Region Alsace', 'Region Auvergne' and CEA, France; German Bundesministerium fur Bildung, Wissenschaft, Forschung und Technologie (BMBF) and the Helmholtz Association; General Secretariat for Research and Technology, Ministry of Development, Greece; National Research, Development and Innovation Office (NKFIH), Hungary; Department of Atomic Energy and Department of Science and Technology of the Government of India; Istituto Nazionale di Fisica Nucleare (INFN) and Centro Fermi-Museo Storico della Fisica e Centro Studi e Ricerche "Enrico Fermi", Italy; Japan Society for the Promotion of Science (JSPS) KAKENHI and MEXT, Japan; Joint Institute for Nuclear Research, Dubna; National Research Foundation of Korea (NRF); Consejo Nacional de Cienca y Tecnologia (CONACYT), Direccion General de Asuntos del Personal Academico(DGAPA), México, Amerique Latine Formation academique-European Commission (ALFA-EC) and the EPLANET Program (European Particle Physics Latin American Network); Stichting voor Fundamenteel Onderzoek der Materie (FOM) and the Nederlandse Organisatie voor Wetenschappelijk Onderzoek (NWO), Netherlands; Research Council of Norway (NFR); National Science Centre, Poland; Ministry of National Education/Institute for Atomic Physics and National Council of Scientific Research in Higher Education (CNCSI-UEFISCDI), Romania; Ministry of Education and Science of Russian Federation, Russian Academy of Sciences, Russian Federal Agency of Atomic Energy, Russian Federal Agency for Science and Innovations and The Russian Foundation for Basic Research; Ministry of Education of Slovakia; Department of Science and Technology, South Africa; Centro de Investigaciones Energeticas, Medioambientales y Tecnologicas (CIEMAT), E-Infrastructure shared between Europe and Latin America (EELA), Ministerio de Economía y Competitividad (MINECO) of Spain, Xunta de Galicia (Consellería de Educación), Centro de Aplicaciones Tecnolgicas y Desarrollo Nuclear (CEADEN), Cubaenergía, Cuba, and IAEA (International Atomic Energy Agency); Swedish Research Council (VR) and Knut \& Alice Wallenberg Foundation (KAW); Ukraine Ministry of Education and Science; United Kingdom Science and Technology Facilities Council (STFC); The United States Department of Energy, the United States National Science Foundation, the State of Texas, and the State of Ohio; Ministry of Science, Education and Sports of Croatia and Unity through Knowledge Fund, Croatia; Council of Scientific and Industrial Research (CSIR), New Delhi, India; Pontificia Universidad Católica del Perú.

Open Access This article is distributed under the terms of the Creative Commons Attribution 4.0 International License (http://creativecomm ons.org/licenses/by/4.0/), which permits unrestricted use, distribution, and reproduction in any medium, provided you give appropriate credit to the original author(s) and the source, provide a link to the Creative Commons license, and indicate if changes were made. Funded by $\mathrm{SCOAP}^{3}$.

\section{References}

1. NA49 Collaboration, S. V. Afanasev et al., Production of $\phi$ mesons in $\mathrm{p}+\mathrm{p}, \mathrm{p}+\mathrm{Pb}$ and central $\mathrm{Pb}+\mathrm{Pb}$ collisions at $\mathrm{E}($ beam $)=158 \mathrm{~A}$ GeV. Phys. Lett. B 491, 59-66 (2000)

2. NA49 Collaboration, C. Alt et al., Energy dependence of $\phi$ meson production in central $\mathrm{Pb}+\mathrm{Pb}$ collisions at $\sqrt{s_{\mathrm{NN}}}=6$ to $17 \mathrm{GeV}$. Phys. Rev. C 78, 044907 (2008). arXiv:0806.1937 [nucl-ex]

3. NA50 Collaboration, M. C. Abreu et al., Evidence for deconfinement of quarks and gluons from the $\mathrm{J} / \psi$ suppression pattern measured in $\mathrm{Pb}+\mathrm{Pb}$ collisions at the CERN SPS. Phys. Lett. B 477, 28-36 (2000)

4. NA50 Collaboration, B. Alessandro et al., A New measurement of $\mathrm{J} / \psi$ suppression in $\mathrm{Pb}-\mathrm{Pb}$ collisions at $158 \mathrm{GeV}$ per nucleon. Eur. Phys. J. C 39, 335-345 (2005). arXiv:hep-ex/0412036 [hep-ex]

5. NA57 Collaboration, F. Antinori et al., Strangeness enhancements at central rapidity in $40 \mathrm{~A} \mathrm{GeV/c} \mathrm{Pb-Pb} \mathrm{collisions.} \mathrm{J.} \mathrm{Phys.} \mathrm{G} \mathrm{37,}$ 045105 (2010). arXiv:1001.1884 [nucl-ex]

6. NA60 Collaboration, R. Arnaldi et al., First measurement of the $\rho$ spectral function in high-energy nuclear collisions. Phys. Rev. Lett. 96, 162302 (2006). arXiv:nucl-ex/0605007 [nucl-ex]

7. NA60 Collaboration, R. Arnaldi et al., Evidence for radial flow of thermal dileptons in high-energy nuclear collisions. Phys. Rev. Lett. 100, 022302 (2008). arXiv:0711.1816 [nucl-ex]

8. NA60 Collaboration, R. Arnaldi et al., A Comparative measurement of $\phi \rightarrow \mathrm{K}^{+} \mathrm{K}^{-}$and $\phi \rightarrow \mu^{+} \mu^{-}$in in-in collisions at the CERN SPS. Phys. Lett. B 699, 325-329 (2011). arXiv:1104.4060 [nucl-ex]

9. NA60 Collaboration, K. Banicz et al., $\phi$ production in inin collisions at 158A GeV. Eur. Phys. J. C 64, 1-18 (2009). arXiv:0906.1102 [hep-ex]

10. WA97 Collaboration, E. Andersen et al., Strangeness enhancement at mid-rapidity in $\mathrm{Pb}-\mathrm{Pb}$ collisions at $158 \mathrm{~A} \mathrm{GeV}$. Phys. Lett. B 449, 401-406 (1999)

11. WA97 Collaboration, F. Antinori et al., Transverse mass spectra of strange and multistrange particles in $\mathrm{Pb}-\mathrm{Pb}$ collisions at $158 \mathrm{~A}$ GeV. Eur. Phys. J. C 14, 633-641 (2000)

12. PHOBOS Collaboration, B.B. Back et al., The PHOBOS perspective on discoveries at RHIC. Nucl. Phys. A 757, 28-101 (2005). arXiv:nucl-ex/0410022 [nucl-ex]

13. STAR Collaboration, J. Adams et al., Experimental and theoretical challenges in the search for the quark gluon plasma: the STAR Collaboration's critical assessment of the evidence from RHIC collisions. Nucl. Phys. A 757, 102-183 (2005). arXiv:nucl-ex/0501009 [nucl-ex]

14. PHENIX Collaboration, K. Adcox et al., Formation of dense partonic matter in relativistic nucleus-nucleus collisions at RHIC: Experimental evaluation by the PHENIX collaboration. Nucl. Phys. A 757, 184-283 (2005). arXiv:nucl-ex/0410003 [nucl-ex]

15. BRAHMS Collaboration, I. Arsene et al., Quark gluon plasma and color glass condensate at RHIC? The Perspective from the BRAHMS experiment. Nucl. Phys. A 757, 1-27 (2005). arXiv:nucl-ex/0410020 [nucl-ex]

16. ALICE Collaboration, K. Aamodt et al., Elliptic flow of charged particles in $\mathrm{Pb}-\mathrm{Pb}$ collisions at $2.76 \mathrm{TeV}$. Phys. Rev. Lett. 105, 252302 (2010). arXiv:1011.3914 [nucl-ex]

17. ALICE Collaboration, K. Aamodt et al., Charged-particle multiplicity density at mid-rapidity in central $\mathrm{Pb}-\mathrm{Pb}$ collisions at 
$\sqrt{s_{\mathrm{NN}}}=2.76$ TeV. Phys. Rev. Lett. 105, 252301 (2010). arXiv:1011.3916 [nucl-ex]

18. ALICE Collaboration, K. Aamodt et al., Centrality dependence of the charged-particle multiplicity density at mid-rapidity in $\mathrm{Pb}-\mathrm{Pb}$ collisions at $\sqrt{s_{\mathrm{NN}}}=2.76 \mathrm{TeV}$. Phys. Rev. Lett. 106, 032301 (2011). arXiv:1012.1657 [nucl-ex]

19. ALICE Collaboration, K. Aamodt et al., Suppression of charged particle production at large transverse momentum in central $\mathrm{Pb}-\mathrm{Pb}$ collisions at $\sqrt{s_{\mathrm{NN}}}=2.76 \mathrm{TeV}$. Phys. Lett. B 696, 30-39 (2011). arXiv:1012.1004 [nucl-ex]

20. ALICE Collaboration, K. Aamodt et al., Two-pion Bose-Einstein correlations in central Pb-Pb collisions at $\sqrt{s_{\mathrm{NN}}}=2.76 \mathrm{TeV}$. Phys. Lett. B 696, 328-337 (2011). arXiv:1012.4035 [nucl-ex]

21. ATLAS Collaboration, G. Aad et al., Observation of a centralitydependent dijet asymmetry in lead-lead collisions at $\sqrt{s_{\mathrm{NN}}}=2.76$ $\mathrm{TeV}$ with the ATLAS detector at the LHC. Phys. Rev. Lett. 105, 252303 (2010). arXiv:1011.6182 [hep-ex]

22. CMS Collaboration, S. Chatrchyan et al., Observation and studies of jet quenching in $\mathrm{PbPb}$ collisions at nucleon-nucleon centerof-mass energy $=2.76$ TeV. Phys. Rev. C 84, 024906 (2011). arXiv:1102.1957 [nucl-ex]

23. STAR Collaboration, J. Adams et al., K(892)* resonance production in $\mathrm{Au}+\mathrm{Au}$ and $\mathrm{p}+\mathrm{p}$ collisions at $\sqrt{s_{\mathrm{NN}}}=200 \mathrm{GeV}$ at STAR. Phys. Rev. C 71, 064902 (2005). arXiv:nucl-ex/0412019 [nucl-ex]

24. STAR Collaboration, B.I. Abelev et al., Strange baryon resonance production in $\sqrt{s_{\mathrm{NN}}}=200 \mathrm{GeV} \mathrm{p}+\mathrm{p}$ and $\mathrm{Au}+\mathrm{Au}$ collisions. Phys. Rev. Lett. 97, 132301 (2006). arXiv:nucl-ex/0604019 [nucl-ex]

25. STAR Collaboration, B.I. Abelev et al., Hadronic resonance production in $\mathrm{d}+\mathrm{Au}$ collisions at $\sqrt{s_{\mathrm{NN}}}=200 \mathrm{GeV}$ at RHIC. Phys. Rev. C 78, 044906 (2008). arXiv:0801.0450 [nucl-ex]

26. STAR Collaboration, B.I. Abelev et al., Measurements of $\phi$ meson production in relativistic heavy-ion collisions at RHIC. Phys. Rev. C 79, 064903 (2009). arXiv:0809.4737 [nucl-ex]

27. STAR Collaboration, M.M. Aggarwal et al., $K^{* 0}$ production in $\mathrm{Cu}+\mathrm{Cu}$ and $\mathrm{Au}+\mathrm{Au}$ collisions at $\sqrt{s_{\mathrm{NN}}}=62.4 \mathrm{GeV}$ and $200 \mathrm{GeV}$. Phys. Rev. C 84, 034909 (2011). arXiv:1006.1961 [nucl-ex]

28. PHENIX Collaboration, A. Adare et al., Nuclear modification factors of $\phi$ mesons in $d+\mathrm{Au}, \mathrm{Cu}+\mathrm{Cu}$ and $\mathrm{Au}+\mathrm{Au}$ collisions at $\sqrt{s_{\mathrm{NN}}}$ = 200 GeV. Phys. Rev. C 83, 024909 (2011). arXiv:1004.3532 [nucl-ex]

29. PHENIX Collaboration, A. Adare et al., $\phi$ meson production in $d+\mathrm{Au}$ collisions at $\sqrt{s_{\mathrm{NN}}}=200 \mathrm{GeV}$. Phys. Rev. C 92(4), 044909 (2015). doi:10.1103/PhysRevC.92.044909. arXiv:1506.08181 [nucl-ex]

30. ALICE Collaboration, B. Abelev et al., Production of $\mathrm{K}^{*}(892)^{0}$ and $\phi(1020)$ in $p p$ collisions at $\sqrt{s}=7 \mathrm{TeV}$. Eur. Phys. J. C 72, 2183 (2012). arXiv:1208.5717 [hep-ex]

31. ATLAS Collaboration, G. Aad et al., The differential production cross section of the $\phi(1020)$ meson in $\sqrt{s}=7 \mathrm{TeV} p p$ collisions measured with the ATLAS detector. Eur. Phys. J. C 74(7), 2895 (2014). arXiv:1402.6162 [hep-ex]

32. LHCb Collaboration, R. Aaij et al., Measurement of the inclusive $\phi$ cross-section in $p p$ collisions at $\sqrt{s}=7 \mathrm{TeV}$. Phys. Lett. B 703, 267-273 (2011). arXiv:1107.3935 [hep-ex]

33. ALICE Collaboration, B.B. Abelev et al., $\mathrm{K}^{*}(892)^{0}$ and $\phi(1020)$ production in $\mathrm{Pb}-\mathrm{Pb}$ collisions at $\sqrt{s_{\mathrm{NN}}}=2.76 \mathrm{TeV}$. Phys. Rev. C 91(2), 024609 (2015). arXiv:1404.0495 [nucl-ex]

34. ALICE Collaboration, J. Adam et al., $\phi$-meson production at forward rapidity in $\mathrm{p}-\mathrm{Pb}$ collisions at $\sqrt{s_{\mathrm{NN}}}=5.02 \mathrm{TeV}$ and in $\mathrm{pp}$ collisions at $\sqrt{s}=2.76 \mathrm{TeV}$. arXiv:1506.09206 [nucl-ex]

35. CMS Collaboration, V. Khachatryan et al., Observation of longrange near-side angular correlations in proton-proton collisions at the LHC. JHEP 09, 091 (2010). arXiv:1009.4122 [hep-ex]

36. CMS Collaboration, S. Chatrchyan et al., Observation of longrange near-side angular correlations in proton-lead collisions at the LHC. Phys. Lett. B 718, 795-814 (2013). arXiv:1210.5482 [nucl-ex]

37. ALICE Collaboration, B. Abelev et al., Long-range angular correlations on the near and away side in $\mathrm{p}-\mathrm{Pb}$ collisions at $\sqrt{s_{\mathrm{NN}}}=5.02$ TeV. Phys. Lett. B 719, 29-41 (2013). arXiv:1212.2001 [nucl-ex]

38. ALICE Collaboration, B.B. Abelev et al., Long-range angular correlations of $\pi, \mathrm{K}$ and $\mathrm{p}$ in $\mathrm{p}-\mathrm{Pb}$ collisions at $\sqrt{s_{\mathrm{NN}}}=5.02 \mathrm{TeV}$. Phys. Lett. B 726, 164-177 (2013). arXiv:1307.3237 [nucl-ex]

39. ALICE Collaboration, B.B. Abelev et al., Multiparticle azimuthal correlations in $\mathrm{p}-\mathrm{Pb}$ and $\mathrm{Pb}-\mathrm{Pb}$ collisions at the CERN large hadron collider. Phys. Rev. C 90(5), 054901 (2014). arXiv:1406.2474 [nucl-ex]

40. P. Bozek, Collective flow in $\mathrm{p}-\mathrm{Pb}$ and d-Pd collisions at $\mathrm{TeV}$ energies. Phys. Rev. C 85, 014911 (2012). arXiv:1112.0915 [hep-ph]

41. P. Bozek, W. Broniowski, Correlations from hydrodynamic flow in p-Pb collisions. Phys. Lett. B. 718, 1557-1561 (2013). arXiv:1211.0845 [nucl-th]

42. ALICE Collaboration, B. Abelev et al., Centrality dependence of $\pi, \mathrm{K}, \mathrm{p}$ production in $\mathrm{Pb}-\mathrm{Pb}$ collisions at $\sqrt{s_{\mathrm{NN}}}=2.76 \mathrm{TeV}$. Phys. Rev. C 88, 044910 (2013). arXiv:1303.0737 [hep-ex]

43. U.W. Heinz, Concepts of heavy ion physics, in 2003 CERN-CLAF School of High-Energy Physics, San Miguel Regla, Mexico, 114 June 2003 (2004), pp. 165-238. http://doc.cern.ch/yellowrep/ CERN-2004-001. arXiv:hep-ph/0407360 [hep-ph]

44. R.J. Fries, B. Muller, C. Nonaka, S.A. Bass, Hadron production in heavy ion collisions: Fragmentation and recombination from a dense parton phase. Phys. Rev. C 68, 044902 (2003). arXiv:nucl-th/0306027 [nucl-th]

45. V. Minissale, F. Scardina, and V. Greco, Hadrons from coalescence plus fragmentation in AA collisions from RHIC to LHC energy. arXiv: 1502.06213 [nucl-th]

46. ALICE Collaboration, K. Aamodt et al., Charged-particle multiplicity measurement in proton-proton collisions at $\sqrt{s}=7$ TeV with ALICE at LHC. Eur. Phys. J. C 68, 345-354 (2010). arXiv: 1004.3514 [hep-ex]

47. ALICE Collaboration, B.B. Abelev et al., Multiplicity dependence of pion, kaon, proton and lambda production in $\mathrm{p}-\mathrm{Pb}$ collisions at $\sqrt{s_{\mathrm{NN}}}=5.02 \mathrm{TeV}$. Phys. Lett. B 728, 25-38 (2014). arXiv:1307.6796 [nucl-ex]

48. ALICE Collaboration, B. Abelev et al., Pseudorapidity density of charged particles in $\mathrm{p}-\mathrm{Pb}$ collisions at $\sqrt{s_{\mathrm{NN}}}=5.02 \mathrm{TeV}$. Phys. Rev. Lett. 110(3), 032301 (2013). arXiv:1210.3615 [nucl-ex]

49. ALICE Collaboration, J. Adam et al., Centrality dependence of particle production in $\mathrm{p}-\mathrm{Pb}$ collisions at $\sqrt{s_{\mathrm{NN}}}=5.02 \mathrm{TeV}$. Phys. Rev. C 91(6), 064905 (2015). arXiv:1412.6828 [nucl-ex]

50. Particle Data Group Collaboration, K. A. Olive et al., Review of particle physics. Chin. Phys. C 38, 090001 (2014)

51. J. Stachel, A. Andronic, P. Braun-Munzinger, K. Redlich, Confronting LHC data with the statistical hadronization model. J. Phys. Conf. Ser. 509, 012019 (2014). arXiv:1311.4662 [nucl-th]

52. M. Bleicher, J. Aichelin, Strange resonance production: Probing chemical and thermal freezeout in relativistic heavy ion collisions. Phys. Lett. B. 530, 81-87 (2002). arXiv:hep-ph/0201123 [hep-ph]

53. A. G. Knospe, C. Markert, K. Werner, J. Steinheimer, M. Bleicher, Hadronic resonance production and interaction in partonic and hadronic matter in EPOS3 with and without the hadronic afterburner UrQMD. Phys. Rev. C 93(1), 014911 (2016). doi:10.1103/ PhysRevC.93.014911. arXiv:1509.07895 [nucl-th]

54. ALICE Collaboration, K. Aamodt et al., The ALICE experiment at the CERN LHC. JINST 3, S08002 (2008)

55. ALICE Collaboration, B.B. Abelev et al., Performance of the ALICE experiment at the CERN LHC. Int. J. Mod. Phys. A 29, 1430044 (2014). arXiv:1402.4476 [nucl-ex]

56. M.L. Miller, K. Reygers, S.J. Sanders, P. Steinberg, Glauber modeling in high energy nuclear collisions. Ann. Rev. Nucl. Part. Sci. 57, 205-243 (2007). arXiv:nucl-ex/0701025 [nucl-ex] 
58. ALICE Collaboration, B. Abelev et al., Centrality determination of $\mathrm{Pb}-\mathrm{Pb}$ collisions at $\sqrt{s_{\mathrm{NN}}}=2.76 \mathrm{TeV}$ with ALICE. Phys. Rev. C 88(4), 044909 (2013). arXiv:1301.4361 [nucl-ex]

59. Axial Field Spectrometer Collaboration, T. Akesson et al., Inclusive vector-meson production in the central region of $\mathrm{pp}$ collisions at $\sqrt{s}=63$ GeV. Nucl. Phys. B 203, 27 (1982). [Erratum: Nucl. Phys. B 229, 541 (1983)]<breakstart $>99</$ breakstart>

60. A. Akindinov et al., Performance of the ALICE time-of-flight detector at the LHC. Eur. Phys. J. Plus 128, 44 (2013)

61. S. Roesler, R. Engel, J. Ranft, The Monte Carlo event generator DPMJET-III, in Advanced Monte Carlo for radiation physics, particle transport simulation and applications. Proceedings, Conference, MC2000, Lisbon, Portugal, October 23-26, 2000, (2000) pp. 1033-1038. http://www-public.slac.stanford. edu/sciDoc/docMeta.aspx?slacPubNumber=SLAC-PUB-8740. arXiv:hep-ph/0012252 [hep-ph]

62. R. Brun, F. Carminati, S. Giani, GEANT3, CERN Programming Library Report (1993)

63. C. Tsallis, Possible generalization of the Boltzmann-Gibbs statistics. J. Stat. Phys. 52, 479-487 (1988)

64. ALICE Collaboration, J. Adam et al., "Measurement of pion, kaon and proton production in proton-proton collisions at $\sqrt{s}=7 \mathrm{TeV}$. Eur. Phys. J. C 75(5), 226 (2015). arXiv:1504.00024 [nucl-ex]
65. STAR Collaboration, B.I. Abelev et al., Strange particle production in $\mathrm{p}+\mathrm{p}$ collisions at $\sqrt{s}=200 \mathrm{GeV}$. Phys. Rev. C 75, 064901 (2007). arXiv:nucl-ex/0607033 [nucl-ex]

66. ALICE Collaboration, B.B. Abelev et al., Production of $\Sigma(1385)^{ \pm}$ and $\Xi(1530)^{0}$ in proton-proton collisions at $\sqrt{s}=7 \mathrm{TeV}$. Eur. Phys. J. C 75(1), 1 (2015). arXiv:1406.3206 [nucl-ex]

67. E. Schnedermann, J. Sollfrank, U.W. Heinz, Thermal phenomenology of hadrons from $200 \mathrm{~A} \mathrm{GeV} \mathrm{S+S} \mathrm{collisions.} \mathrm{Phys.} \mathrm{Rev.} \mathrm{C.} \mathrm{48,}$ 2462-2475 (1993). arXiv:nucl-th/9307020 [nucl-th]

68. ALICE Collaboration, J. Adam et al., Multi-strange baryon production in p-Pb collisions at $\sqrt{s_{\mathrm{NN}}}=5.02 \mathrm{TeV}$. arXiv: 1512.07227 [nucl-ex]

69. ALICE Collaboration, J. Adam et al., Two-pion femtoscopy in $\mathrm{p}-\mathrm{Pb}$ collisions at $\sqrt{s_{\mathrm{NN}}}=5.02 \mathrm{TeV}$. Phys. Rev. C 91, 034906 (2015). arXiv:1502.00559 [nucl-ex]

70. ALICE Collaboration, B.B. Abelev et al., Multiplicity dependence of the average transverse momentum in $\mathrm{pp}, \mathrm{p}-\mathrm{Pb}$, and $\mathrm{Pb}-$ Pb collisions at the LHC. Phys. Lett. B 727, 371-380 (2013). arXiv:1307.1094 [nucl-ex]

\section{ALICE Collaboration}

J. Adam ${ }^{40}$, D. Adamová ${ }^{84}$, M. M. Aggarwal ${ }^{88}$, G. Aglieri Rinella ${ }^{36}$, M. Agnello ${ }^{110}$, N. Agrawal ${ }^{48}$, Z. Ahammed ${ }^{132}$, S. Ahmad ${ }^{19}, \quad$ S. U. Ahn ${ }^{68}, \quad$ S. Aiola ${ }^{136}$, A. Akindinov ${ }^{58}, \quad$ S. N. Alam ${ }^{132}$, D. Aleksandrov ${ }^{80}, \quad$ B. Alessandro ${ }^{110}$, D. Alexandre ${ }^{101}$, R. Alfaro Molina ${ }^{64}$, A. Alici ${ }^{12,104}$, A. Alkin ${ }^{3}$, J. R. M. Almaraz ${ }^{119}$, J. Alme ${ }^{38}$, T. Alt ${ }^{43}$, S. Altinpinar ${ }^{18}$, I. Altsybeev ${ }^{131}$, C. Alves Garcia Prado ${ }^{120}$, C. Andrei ${ }^{78}$, A. Andronic ${ }^{97}$, V. Anguelov ${ }^{94}$, T. Antičicic ${ }^{98}$, F. Antinori ${ }^{107}$, P. Antonioli ${ }^{104}$, L. Aphecetche ${ }^{113}$, H. Appelshäuser ${ }^{53}$, S. Arcelli ${ }^{28}$, R. Arnaldi ${ }^{110}$, O. W. Arnold ${ }^{37,93}$, I. C. Arsene ${ }^{22}$, $\begin{array}{ll}\text { M. Arslandok } & 53\end{array}$ B. Audurier ${ }^{13}$, A. Augustinus ${ }^{36}, \quad$ R. Averbeck ${ }^{97}, \quad$ M. D. Azmi ${ }^{19}$, A. Badalà ${ }^{106}$, Y. W. Baek ${ }^{67}$, S. Bagnasco ${ }^{110}$, R. Bailhache ${ }^{53}$, R. Bala ${ }^{91}$, S. Balasubramanian ${ }^{136}$, A. Baldisseri ${ }^{15}$, R. C. Baral ${ }^{61}$, A. M. Barbano ${ }^{27}$, R. Barbera ${ }^{29}$ ，F. Barile ${ }^{33}$ ，G. G. Barnaföldi ${ }^{135}$ ， L. S. Barnby ${ }^{101}$ ，V. Barret ${ }^{70}$ ，P. Bartalini ${ }^{7}$ ，K. Barth ${ }^{36}$ ， J. Bartke ${ }^{117}$, E. Bartsch ${ }^{53}$, M. Basile ${ }^{28}$, N. Bastid ${ }^{70}$, S. Basu ${ }^{132}$, B. Bathen ${ }^{54}$, G. Batigne ${ }^{113}$, A. Batista Camejo ${ }^{70}$, B. Batyunya ${ }^{66}$, P. C. Batzing ${ }^{22}$, I. G. Bearden ${ }^{81}$, H. Beck ${ }^{53}$, C. Bedda ${ }^{110}$, N. K. Behera ${ }^{50}$, I. Belikov ${ }^{55}$, F. Bellini2 ${ }^{28}$, H. Bello Martinez ${ }^{2}$, R. Bellwied ${ }^{122}$, R. Belmont ${ }^{134}$, E. Belmont-Moreno ${ }^{64}$, V. Belyaev ${ }^{75}$, P. Benacek ${ }^{84}$, G. Bencedi ${ }^{135}$, S. Beole ${ }^{27}$, I. Berceanu $^{78}$, A. Bercuci ${ }^{78}$, Y. Berdnikov ${ }^{86}$, D. Berenyi ${ }^{135}$, R. A. Bertens ${ }^{57}$, D. Berzano ${ }^{36}$, L. Betev ${ }^{36}$, A. Bhasin ${ }^{91}$, I. R. Bhat ${ }^{91}$, A. K. Bhati ${ }^{88}$, B. Bhattacharjee ${ }^{45}$, J. Bhom ${ }^{128}$, L. Bianchi ${ }^{122}$, N. Bianchi ${ }^{72}$, C. Bianchin ${ }^{57,134}$, J. Bielčík ${ }^{40}$, J. Bielčíková ${ }^{84}$, A. Bilandzic ${ }^{37,81,93}$, G. Biro ${ }^{135}$, R. Biswas ${ }^{4,79}$, S. Biswas ${ }^{79}$, S. Bjelogrlic ${ }^{57}$, J. T. Blair ${ }^{118}$, D. Blau ${ }^{80}$, C. Blume ${ }^{53}$, F. Bock ${ }^{74,94}$, A. Bogdanov ${ }^{75}$, H. Bøggild ${ }^{81}$, L. Boldizsár ${ }^{135}$, M. Bombara ${ }^{41}$, J. Book ${ }^{53}$, H. Borel ${ }^{15}$, A. Borissov ${ }^{96}$, M. Borri ${ }^{83,124}$, F. Bossú ${ }^{65}$, E. Botta ${ }^{27}$, C. Bourjau ${ }^{81}$, P. Braun-Munzinger ${ }^{97}$, M. Bregant ${ }^{120}$, T. Breitner ${ }^{52}$, T. A. Broker ${ }^{53}$, T. A. Browning ${ }^{95}$, M. Broz ${ }^{40}$, E. J. Brucken ${ }^{46}$, E. Bruna ${ }^{110}$, G. E. Bruno ${ }^{33}$, D. Budnikov ${ }^{99}$, H. Buesching ${ }^{53}$, S. Bufalino 27,36 , P. Buncic ${ }^{36}$, O. Busch ${ }^{94,128}$, Z. Buthelezi ${ }^{65}$, J. B. Butt ${ }^{16}$, J. T. Buxton ${ }^{20}$, D. Caffarri ${ }^{36}$, X. $\mathrm{Cai}^{7}$, H. Caines ${ }^{136}$, L. Calero Diaz ${ }^{72}$, A. Caliva ${ }^{57}$, E. Calvo Villar ${ }^{102}$, P. Camerini ${ }^{26}$, F. Carena ${ }^{36}$, W. Carena ${ }^{36}$, F. Carnesecchi ${ }^{28}$, J. Castillo Castellanos ${ }^{15}$, A. J. Castro ${ }^{125}$, E. A. R. Casula ${ }^{25}$, C. Ceballos Sanchez ${ }^{9}$ ， P. Cerello ${ }^{110}$, J. Cerkala ${ }^{115}$, B. Chang ${ }^{123}$, S. Chapeland ${ }^{36}$, M. Chartier ${ }^{124}$, J. L. Charvet ${ }^{15}$, S. Chattopadhyay ${ }^{132}$, S. Chattopadhyay ${ }^{100}$, A. Chauvin ${ }^{37,93}$, V. Chelnokov ${ }^{3}$, M. Cherney ${ }^{87}$, C. Cheshkov ${ }^{130}$, B. Cheynis ${ }^{130}$, V. Chibante Barroso ${ }^{36}$, D. D. Chinellato ${ }^{121}$, S. Cho ${ }^{50}$, P. Chochula ${ }^{36}$, K. Choi ${ }^{96}$, M. Chojnacki ${ }^{81}$, S. Choudhury ${ }^{132}$, P. Christakoglou ${ }^{82}$, C. H. Christensen ${ }^{81}$, P. Christiansen ${ }^{34}$, T. Chujo ${ }^{128}$, S. U. Chung ${ }^{96}$, C. Cicalo ${ }^{105}$, L. Cifarelli ${ }^{12,28}$, F. Cindolo ${ }^{104}$, J. Cleymans ${ }^{90}$, F. Colamaria ${ }^{33}$, D. Colella ${ }^{36,59}$, A. Collu ${ }^{25,74}$, M. Colocci ${ }^{28}$, G. Conesa Balbastre ${ }^{71}$, Z. Conesa del Valle ${ }^{51}$, M. E. Connors ${ }^{136, a}$, J. G. Contreras ${ }^{40}$, T. M. Cormier ${ }^{85}$, Y. Corrales Morales ${ }^{110}$, I. Cortés Maldonado ${ }^{2}$, P. Cortese ${ }^{32}$, M. R. Cosentino ${ }^{120}$, F. Costa ${ }^{36}$, P. Crochet ${ }^{70}$, R. Cruz Albino ${ }^{11}$, E. Cuautle ${ }^{63}$, L. Cunqueiro ${ }^{36,54}$, T. Dahms ${ }^{37,93}$, A. Dainese ${ }^{107}$, M. C. Danisch ${ }^{94}$, A. Danu ${ }^{62}$, D. Das ${ }^{100}$,

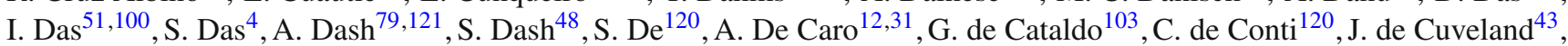
A. De Falco ${ }^{25}$ ，D. De Gruttola ${ }^{12,31}$ ，N. De Marco ${ }^{110}$ ， S. De Pasquale ${ }^{31}$ ，A. Deisting ${ }^{94,97}$ ，A. Deloff ${ }^{77}$, E. Dénes ${ }^{135, a}$, C. Deplano ${ }^{82}$, P. Dhankher ${ }^{48}$, D. Di Bari ${ }^{33}$, A. Di Mauro ${ }^{36}, \quad$ P. Di Nezza ${ }^{72}, \quad$ M. A. Diaz Corchero ${ }^{10}, \quad$ T. Dietel ${ }^{90}$, 
P. Dillenseger ${ }^{53}$, R. Divià ${ }^{36}$ ， Ø. Djuvsland ${ }^{18}$ ，A. Dobrin ${ }^{62,82}$ ，D. Domenicis Gimenez ${ }^{120}$ ，B. Dönigus ${ }^{53}$ ， O. Dordic ${ }^{22}$, T. Drozhzhova ${ }^{53}$, A. K. Dubey ${ }^{132}$, A. Dubla ${ }^{57}$, L. Ducroux ${ }^{130}$, P. Dupieux ${ }^{70}$, R. J. Ehlers ${ }^{136}$, D. Elia ${ }^{103}$, E. Endress ${ }^{102}$, H. Engel ${ }^{52}$, E. Epple ${ }^{136}$, B. Erazmus ${ }^{113}$, I. Erdemir ${ }^{53}$, F. Erhardt ${ }^{129}$, B. Espagnon ${ }^{51}$, M. Estienne ${ }^{113}$, S. Esumi ${ }^{128}$, J. Eum ${ }^{96}$, D. Evans ${ }^{101}$, S. Evdokimov ${ }^{111}$, G. Eyyubova ${ }^{40}$, L. Fabbietti ${ }^{37,93}$, D. Fabris ${ }^{107}$, J. Faivre ${ }^{71}$, A. Fantoni ${ }^{72}$, M. Fasel ${ }^{74}$, L. Feldkamp ${ }^{54}$, A. Feliciello ${ }^{110}$, G. Feofilov ${ }^{131}$, J. Ferencei ${ }^{84}$, A. Fernández Téllez ${ }^{2}$, E. G. Ferreiro ${ }^{17}$, A. Ferretti ${ }^{27}$, A. Festanti ${ }^{30}$, V. J. G. Feuillard ${ }^{15,70}$, J. Figiel ${ }^{117}$, M. A. S. Figueredo ${ }^{120,124}$, S. Filchagin ${ }^{99}$, D. Finogeev ${ }^{56}$, F. M. Fionda ${ }^{25}$, E. M. Fiore ${ }^{33}$, M. G. Fleck ${ }^{94}$, M. Floris ${ }^{36}$, S. Foertsch ${ }^{65}$, P. Foka ${ }^{97}$, S. Fokin ${ }^{80}$, E. Fragiacomo ${ }^{109}$, A. Francescon ${ }^{30,36}$, U. Frankenfeld ${ }^{97}$, G. G. Fronze ${ }^{27}$, U. Fuchs ${ }^{36}$, C. Furget ${ }^{71}$, A. Furs ${ }^{56}$, M. Fusco Girard ${ }^{31}$, J. J. Gaardhøje ${ }^{81}$, M. Gagliardi ${ }^{27}$, A. M. Gago ${ }^{102}$, M. Gallio ${ }^{27}$, D. R. Gangadharan ${ }^{74}$, P. Ganoti ${ }^{89}$, C. Gao ${ }^{7}$, C. Garabatos ${ }^{97}$, E. Garcia-Solis ${ }^{13}$, C. Gargiulo ${ }^{36}$, P. Gasik ${ }^{37,93}$, E. F. Gauger ${ }^{118}$, M. Germain ${ }^{113}$, A. Gheata ${ }^{36}$, M. Gheata ${ }^{36,62}$, P. Ghosh ${ }^{132}$, S. K. Ghosh ${ }^{4}$, P. Gianotti ${ }^{72}$, P. Giubellino ${ }^{36,110}$ ，P. Giubilato ${ }^{30}$, E. Gladysz-Dziadus ${ }^{117}$ ， P. Glässel ${ }^{94}$ ，D. M. Goméz Coral ${ }^{64}$, A. Gomez Ramirez ${ }^{52}$, V. Gonzalez ${ }^{10}$, P. González-Zamora ${ }^{10}$, S. Gorbunov ${ }^{43}$, L. Görlich ${ }^{117}$, S. Gotovac ${ }^{116}$, V. Grabski ${ }^{64}$, O. A. Grachov ${ }^{136}$, L. K. Graczykowski ${ }^{133}$, K. L. Graham ${ }^{101}$, A. Grelli ${ }^{57}$, A. Grigoras ${ }^{36}$, C. Grigoras ${ }^{36}$, V. Grigoriev ${ }^{75}$, A. Grigoryan ${ }^{1}$, S. Grigoryan ${ }^{66}$, B. Grinyov ${ }^{3}$, N. Grion ${ }^{109}$, J. M. Gronefeld ${ }^{97}$, J. F. Grosse-Oetringhaus ${ }^{36}$, J.-Y. Grossiord ${ }^{130}$, R. Grosso ${ }^{97}$, F. Guber ${ }^{56}$, R. Guernane ${ }^{71}$, B. Guerzoni ${ }^{28}$, K. Gulbrandsen ${ }^{81}$, T. Gunji ${ }^{127}$, A. Gupta ${ }^{91}$, R. Gupta ${ }^{91}$, R. Haake ${ }^{54}$, Ø. Haaland ${ }^{18}$, C. Hadjidakis ${ }^{51}$, M. Haiduc ${ }^{62}$, H. Hamagaki ${ }^{127}$, G. Hamar ${ }^{135}$, J. C. Hamon ${ }^{55}$, J. W. Harris ${ }^{136}$, A. Harton ${ }^{13}$, D. Hatzifotiadou ${ }^{104}$, S. Hayashi ${ }^{127}$, S. T. Heckel ${ }^{53}$, E. Hellbär ${ }^{53}$, H. Helstrup ${ }^{38}$, A. Herghelegiu ${ }^{78}$, G. Herrera Corral ${ }^{11}$,

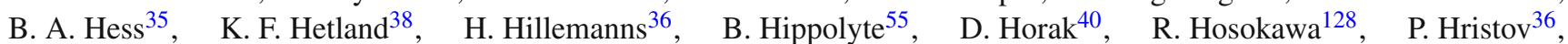
M. Huang ${ }^{18}$, T. J. Humanic ${ }^{20}$, N. Hussain ${ }^{45}$, T. Hussain ${ }^{19}$, D. Hutter ${ }^{43}$, D. S. Hwang ${ }^{21}$, R. Ilkaev99, M. Inaba ${ }^{128}$, E. Incani ${ }^{25}$, M. Ippolitov ${ }^{75,80}$, M. Irfan ${ }^{19}$, M. Ivanov ${ }^{97}$, V. Ivanov ${ }^{86}$, V. Izucheev ${ }^{111}$ ， N. Jacazio ${ }^{28}$, P. M. Jacobs ${ }^{74}$, M. B. Jadhav ${ }^{48}$ ，S. Jadlovska ${ }^{115}$ ，J. Jadlovsky ${ }^{59,115}$ ，C. Jahnke ${ }^{120}$ ， M. J. Jakubowska ${ }^{133}$ ， H. J. Jang ${ }^{68}$ ， M. A. Janik ${ }^{133}$, P. H. S. Y. Jayarathna ${ }^{122}$ ，C. Jena ${ }^{30}$ ，S. Jena ${ }^{122}$ ， R. T. Jimenez Bustamante ${ }^{97}$ ，P. G. Jones ${ }^{101}$ ，A. Jusko ${ }^{101}$, P. Kalinak ${ }^{59}$, A. Kalweit ${ }^{36}$, J. Kamin ${ }^{53}$, J. H. Kang ${ }^{137}$, V. Kaplin ${ }^{75}$, S. Karr ${ }^{132}$, A. Karasu Uysal ${ }^{69}$, O. Karavichev ${ }^{56}$, T. Karavicheva ${ }^{56}$, L. Karayan ${ }^{94,97}$, E. Karpechev ${ }^{56}$, U. Kebschull ${ }^{52}$, R. Keidel ${ }^{138}$, D. L. D. Keijdener ${ }^{57}$, M. Keil ${ }^{36}$, M. Mohisin Khan ${ }^{19, b}$, P. Khan ${ }^{100}$, S. A. Khan ${ }^{132}$, A. Khanzadeev ${ }^{86}$, Y. Kharlov ${ }^{111}$, B. Kileng ${ }^{38}$, D. W. Kim ${ }^{44}$, D. J. Kim ${ }^{123}$, D. Kim ${ }^{137}$, H. Kim ${ }^{137}$,

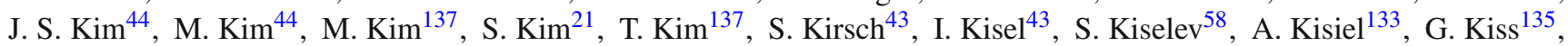
J. L. Klay ${ }^{6}$, C. Klein ${ }^{53}$, J. Klein ${ }^{36}$, C. Klein-Bösing ${ }^{54}$, S. Klewinn4, A. Kluge ${ }^{36}$, M. L. Knichel ${ }^{94}$, A. G. Knospe ${ }^{118,122}$, C. Kobdaj ${ }^{114}$, M. Kofarago ${ }^{36}$, T. Kollegger ${ }^{97}$, A. Kolojvari ${ }^{131}$, V. Kondratiev ${ }^{131}$, N. Kondratyeva ${ }^{75}$, E. Kondratyuk ${ }^{111}$, A. Konevskikh ${ }^{56}$, M. Kopcik ${ }^{115}$, P. Kostarakis ${ }^{89}$, M. Kour91, C. Kouzinopoulos ${ }^{36}$, O. Kovalenko ${ }^{77}$, V. Kovalenko ${ }^{131}$, M. Kowalski117, G. Koyithatta Meethaleveedu ${ }^{48}$, I. Králik ${ }^{59}$, A. Kravčáková41, M. Kretz ${ }^{43}$, M. Krivda ${ }^{59,101}$, F. Krizek ${ }^{84}$, E. Kryshen ${ }^{36,86}$, M. Krzewicki³ ${ }^{43}$ A. M. Kubera ${ }^{20}$, V. Kučera ${ }^{84}$, C. Kuhn5, P. G. Kuijer ${ }^{82}$, A. Kumar ${ }^{91}$, J. Kumar ${ }^{48}$, L. $K_{u m a r}^{88}$, S. Kumar ${ }^{48}$, P. Kurashvili ${ }^{77}$, A. Kurepin' ${ }^{56}$, A. B. Kurepin' ${ }^{56}$, A. Kuryakin99, M. J. Kweon ${ }^{50}$, Y. Kwon ${ }^{137}$, S. L. La Pointe ${ }^{110}$, P. La Rocca ${ }^{29}$, P. Ladron de Guevara ${ }^{11}$, C. Lagana Fernandes ${ }^{120}$, I. Lakomov ${ }^{36}$, R. Langoy ${ }^{42}$, C. Lara $^{52}$, A. Lardeux ${ }^{15}$, A. Lattuca ${ }^{27}$, E. Laudi ${ }^{36}$, R. Lea ${ }^{26}$, L. Leardini ${ }^{94}$, G. R. Lee ${ }^{101}$, S. Lee ${ }^{137}$, F. Lehas ${ }^{82}$, R. C. Lemmon ${ }^{83}$, V. Lenti ${ }^{103}$, E. Leogrande ${ }^{57}$, I. León Monzón ${ }^{119}$, H. León Vargas ${ }^{64}$, M. Leoncino ${ }^{27}$, P. Lévai ${ }^{135}, \quad$ S. Li ${ }^{7,70}, \quad X$. Li $^{14}$, J. Lien ${ }^{42}$, R. Lietava ${ }^{101}$, S. Lindal ${ }^{22}$, V. Lindenstruth ${ }^{43}$, C. Lippmann ${ }^{97}$, M. A. Lisa ${ }^{20}$, H. M. Ljunggren ${ }^{34}$, D. F. Lodato ${ }^{57}$, P. I. Loenne ${ }^{18}$, V. Loginov ${ }^{75}$, C. Loizides ${ }^{74}$, X. Lopez ${ }^{70}$, E. López Torres ${ }^{9}$, A. Lowe ${ }^{135}$, P. Luettig ${ }^{53}$, M. Lunardon ${ }^{30}$, G. Luparello ${ }^{26}$, T. H. Lutz ${ }^{136}$, A. Maevskaya ${ }^{56}$, M. Mager $^{36}$, S. Mahajan ${ }^{91}$, S. M. Mahmood ${ }^{22}$, A. Maire ${ }^{55}$, R. D. Majka ${ }^{136}$, M. Malaev ${ }^{86}$, I. Maldonado Cervantes ${ }^{63}$, L. Malinina ${ }^{66, \mathrm{c}}$, D. Mal'Kevich ${ }^{58}$, P. Malzacher ${ }^{97}$, A. Mamonov ${ }^{99}$, V. Manko ${ }^{80}$, F. Manso ${ }^{70}$, V. Manzari ${ }^{36,103}$, M. Marchisone ${ }^{27,65,126}$, J. Mareš ${ }^{60}$, G. V. Margagliotti ${ }^{26}$, A. Margotti ${ }^{104}$, J. Margutti ${ }^{57}$, A. Marín ${ }^{97}$, C. Markert ${ }^{118}$, M. Marquard ${ }^{53}$, N. A. Martinn ${ }^{97}$, J. Martin Blanco ${ }^{113}$, P. Martinengo ${ }^{36}$, M. I. Martínez ${ }^{2}$, G. Martínez García ${ }^{113}$, M. Martinez Pedreira ${ }^{36}$, A. Mas ${ }^{120}$, S. Masciocchi ${ }^{97}$, M. Masera ${ }^{27}$, A. Masoni ${ }^{105}$, L. Massacrier ${ }^{113}$, A. Mastroserio ${ }^{33}$, A. Matyja ${ }^{117}$, C. Mayer ${ }^{36,117}$, J. Mazer ${ }^{125}, \quad$ M. A. Mazzoni ${ }^{108}, \quad$ D. Mcdonald ${ }^{122}, \quad$ F. Meddi ${ }^{24}$, Y. Melikyan ${ }^{75}$, A. Menchaca-Rocha ${ }^{64}$, E. Meninno ${ }^{31}$, J. Mercado Pérez ${ }^{94}$, M. Meres ${ }^{39}$, Y. Miake ${ }^{128}$, M. M. Mieskolainen ${ }^{46}$, K. Mikhaylov ${ }^{58,66}$, L. Milano ${ }^{36,74}$, J. Milosevic ${ }^{22}$, L. M. Minervini ${ }^{23,103}$, A. Mischke ${ }^{57}$, A. N. Mishra ${ }^{49}$, D. Miśkowiec ${ }^{97}$, J. Mitra ${ }^{132}$, C. M. Mitu ${ }^{62}$, N. Mohammadi ${ }^{57}$, B. Mohanty ${ }^{79,132}$, L. Molnar ${ }^{55,113}$, L. Montaño Zetina ${ }^{11}$, E. Montes ${ }^{10}$, D. A. Moreira De Godoy ${ }^{54,113}$, L. A. P. Moreno ${ }^{2}, \quad$ S. Moretto ${ }^{30}, \quad$ A. Morreale ${ }^{113}$, A. Morsch ${ }^{36}, \quad$ V. Muccifora ${ }^{72}$, E. Mudnic ${ }^{116}$, D. Mühlheim ${ }^{54}$, S. Muhuri ${ }^{132}$, M. Mukherjee ${ }^{132}$, J. D. Mulligan ${ }^{136}$, M. G. Munhoz ${ }^{120}$, R. H. Munzer ${ }^{37,93}$, H. Murakami ${ }^{127}$, S. Murray ${ }^{65}$, L. Musa ${ }^{36}$, J. Musinsky ${ }^{59}$, B. Naik ${ }^{48}$, R. Nair ${ }^{77}$, B. K. Nandi ${ }^{48}$, R. Nania ${ }^{104}$, E. Nappi ${ }^{103}$, M. U. Naru ${ }^{16}$, H. Natal da Luz ${ }^{120}$, C. Nattrass ${ }^{125}$, S. R. Navarro ${ }^{2}$, K. Nayak ${ }^{79}$, R. Nayak ${ }^{84}$, T. K. Nayak ${ }^{132}$, S. Nazarenko ${ }^{99}$, A. Nedosekin ${ }^{58}$, L. Nellen ${ }^{63}$, F. Ng ${ }^{122}$, M. Nicassio ${ }^{97}$, M. Niculescu ${ }^{62}$, J. Niedziela ${ }^{36}$, B. S. Nielsen ${ }^{81}$, S. Nikolaev ${ }^{80}$, S. Nikulin ${ }^{80}$, V. Nikulin ${ }^{86}$, F. Noferini ${ }^{12,104}$, P. Nomokonov ${ }^{66}$, G. Nooren ${ }^{57}$, J. C. C. Noris ${ }^{2}$, J. Norman ${ }^{124}$, A. Nyanin ${ }^{80}$, J. Nystrand ${ }^{18}$, H. Oeschler ${ }^{94}$, S. Oh ${ }^{136}$, S. K. Oh ${ }^{67}$, A. Ohlson ${ }^{36}$, A. Okatan ${ }^{69}$, T. Okubo ${ }^{47}$, L. Olah ${ }^{135}$, J. Oleniacz ${ }^{133}$, 
A. C. Oliveira Da Silva ${ }^{120}$ ， M. H. Oliver ${ }^{136}$ ， J. Onderwaater ${ }^{97}$ ， C. Oppedisano ${ }^{110}$ ， R. Orava ${ }^{46}$ ，A. Ortiz Velasquez ${ }^{63}$, A. Oskarsson ${ }^{34}$, J. Otwinowski ${ }^{117}$, K. Oyama ${ }^{76,94}$, M. Ozdemir ${ }^{53}$, Y. Pachmayer ${ }^{94}$, P. Pagano ${ }^{31}$, G. Paić63 ${ }^{63}$ S. K. Pal ${ }^{132}$, J. Pan ${ }^{134}$ ， A. K. Pandey ${ }^{48}$ ， V. Papikyan ${ }^{1}$ ， G. S. Pappalardo ${ }^{106}$ ， P. Pareek ${ }^{49}$ ，W. J. Park ${ }^{97}$ ， S. Parmar ${ }^{88}$ ，A. Passfeld ${ }^{54}$, V. Paticchio ${ }^{103}$, R. N. Patra ${ }^{132}$, B. Paul ${ }^{100}, \quad$ H. Pei ${ }^{7}, \quad$ T. Peitzmann ${ }^{57}, \quad$ H. Pereira Da Costa ${ }^{15}$, D. Peresunko ${ }^{75,80}$, C. E. Pérez Lara ${ }^{82}$, E. Perez Lezama ${ }^{53}$, V. Peskov ${ }^{53}$, Y. Pestov ${ }^{5}$, V. Petráček ${ }^{40}$, V. Petrov ${ }^{111}$, M. Petrovici ${ }^{78}$, C. Petta ${ }^{29}$, S. Piano ${ }^{109}$, M. Pikna ${ }^{39}$, P. Pillot ${ }^{113}$, L. O. D. L. Pimentel ${ }^{81}$, O. Pinazza ${ }^{36,104}$, L. Pinsky ${ }^{122}$, D. B. Piyarathna ${ }^{122}$, $\begin{array}{llll}\text { M. Płoskoń } & 74 & \text { M. Planinic } & 129\end{array} \quad$ J. Pluta ${ }^{133}$ ， S. Pochybova ${ }^{135}$, P. L. M. Podesta-Lerma ${ }^{119}, \quad$ M. G. Poghosyan ${ }^{85,87}$, B. Polichtchouk ${ }^{111}$ ， N. Poljak ${ }^{129}$ ， W. Poonsawat ${ }^{114}$, A. Pop ${ }^{78}$ ， S. Porteboeuf-Houssais ${ }^{70}$, J. Porter ${ }^{74}$, J. Pospisil ${ }^{84}$, S. K. Prasad ${ }^{4}$, R. Preghenella ${ }^{36,104}$,F. Prino ${ }^{110}$, C. A. Pruneau ${ }^{134}$, I. Pshenichnov ${ }^{56}$, M. Puccio $^{27}$, G. Puddu ${ }^{25}$,P. Pujahari ${ }^{134}$, V. Punin ${ }^{99}$, J. Putschke ${ }^{134}$, H. Qvigstad ${ }^{22}$, A. Rachevski ${ }^{109}$, S. Raha ${ }^{4}$, S. Rajput ${ }^{91}$, J. Rak ${ }^{123}$, A. Rakotozafindrabe ${ }^{15}$, L. Ramello ${ }^{32}$, F. Rami ${ }^{55}$, R. Raniwala ${ }^{92}$, S. Raniwala ${ }^{92}$, S. S. Räsänen ${ }^{46}$, B. T. Rascanu ${ }^{53}$, D. Rathee ${ }^{88}$, K. F. Read ${ }^{85,125}$, K. Redlich ${ }^{77}$, R. J. Reed ${ }^{134}$, A. Rehman ${ }^{18}$, P. Reichelt ${ }^{53}$, F. Reidt ${ }^{36,94}$, X. Ren ${ }^{7}$, R. Renfordt ${ }^{53}$, A. R. Reolon ${ }^{72}$, A. Reshetin ${ }^{56}$, J.-P. Revol ${ }^{12}$, K. Reygers ${ }^{94}$, V. Riabov ${ }^{86}$, R. A. Ricci ${ }^{73}$, T. Richert ${ }^{34}$, M. Richter ${ }^{22}$, P. Riedler ${ }^{36}$, W. Riegler ${ }^{36}$, F. Riggi ${ }^{29}$ ， C. Ristea ${ }^{62}$, E. Rocco ${ }^{57}$, M. Rodríguez Cahuantzi ${ }^{2}, 11$, A. Rodriguez Manso ${ }^{82}$, K. Røed ${ }^{22}$, E. Rogochaya ${ }^{66}$, D. Rohr ${ }^{43}$, D. Röhrich ${ }^{18}$, R. Romita ${ }^{124}$,F. Ronchetti ${ }^{36,72}$, L. Ronflette ${ }^{113}$, P. Rosnet ${ }^{70}$, A. Rossi ${ }^{30,36}$, F. Roukoutakis ${ }^{89}$, A. Roy ${ }^{49}$, C. Roy ${ }^{55}$, P. Roy ${ }^{100}$, A. J. Rubio Montero ${ }^{10}$, R. Rui ${ }^{26}$, R. Russo ${ }^{27}$, E. Ryabinkin ${ }^{80}$, Y. Ryabov ${ }^{86}$, A. Rybicki1 ${ }^{117}$ ，S. Sadovsky ${ }^{111}$ ， K. Šafařík ${ }^{36}$ ， B. Sahlmuller ${ }^{53}$ ，P. Sahoo ${ }^{49}$ ， R. Sahoo ${ }^{49}$ ，S. Sahoo ${ }^{61 ， \quad P . ~ K . ~ S a h u ~}{ }^{61}$, J. Saini ${ }^{132}$ ，S. Sakai ${ }^{74}$, M. A. Saleh ${ }^{134}$ ， J. Salzwedel ${ }^{20}$ ，S. Sambyal ${ }^{91}$ ， V. Samsonov ${ }^{86}$, L. Šándor ${ }^{59}$, A. Sandoval ${ }^{64}$, M. Sano ${ }^{128}$, D. Sarkar ${ }^{132}$, P. Sarma ${ }^{45}$, E. Scapparone ${ }^{104}$, F. Scarlassara ${ }^{30}$, C. Schiaua ${ }^{78}$, R. Schicker ${ }^{94}$, C. Schmidt ${ }^{97}$, H. R. Schmidt ${ }^{35}$ ， S. Schuchmann ${ }^{53}$ ， J. Schukraft ${ }^{36}, \quad$ M. Schulc ${ }^{40}$ ， T. Schuster ${ }^{136}$ ， Y. Schutz ${ }^{36,113}$ ， K. Schwarz ${ }^{97}$, K. Schweda ${ }^{97}$, G. Scioli ${ }^{28}$, E. Scomparin ${ }^{110}$, R. Scott ${ }^{125}$, M. Šefčík ${ }^{41}$, J. E. Seger ${ }^{87}$, Y. Sekiguchi ${ }^{127}$, D. Sekihata ${ }^{47}$, I. Selyuzhenkov $^{97}$, K. Senosi ${ }^{65}$, S. Senyukov ${ }^{3,36}$, E. Serradilla ${ }^{10,64}$, A. Sevcenco ${ }^{62}$, A. Shabanov ${ }^{56}$, A. Shabetai ${ }^{113}$, O. Shadura ${ }^{3}$, R. Shahoyan ${ }^{36}$, A. Shangaraev ${ }^{111}$, A. Sharma ${ }^{91}$, M. Sharma ${ }^{91}$, M. Sharma ${ }^{91}$, N. Sharma ${ }^{125}$, K. Shigaki ${ }^{47}$, K. Shtejer ${ }^{9,27}$, Y. Sibiriak ${ }^{80}$ ， S. Siddhanta ${ }^{105}$ ， K. M. Sielewicz ${ }^{36}$ ， T. Siemiarczuk ${ }^{77}$ ， D. Silvermyr ${ }^{34}$ ， C. Silvestre ${ }^{71}$, G. Simatovic ${ }^{129}$, G. Simonetti ${ }^{36}$, R. Singaraju ${ }^{132}$, R. Singh $^{79}$, S. Singha ${ }^{79,132}$, V. Singhal ${ }^{132}$, B. C. Sinha ${ }^{132}$, T. Sinha ${ }^{100}$, B. $\operatorname{Sitar}^{39}$, M. Sitta ${ }^{32}$, T. B. Skaali ${ }^{22}$, M. Slupecki ${ }^{123}$, N. Smirnov ${ }^{136}$, R. J. M. Snellings ${ }^{57}$, T. W. Snellman ${ }^{123}$, C. Søgaard ${ }^{34}$, J. Song ${ }^{96}$, M. Song ${ }^{137}$, Z. Song ${ }^{7}$, F. Soramel ${ }^{30}$, S. Sorensen ${ }^{125}$, R. D. de Souza ${ }^{121}$, F. Sozzi ${ }^{97}$, M. Spacek ${ }^{40}$, E. Spiriti ${ }^{72}$, I. Sputowska ${ }^{117}$, M. Spyropoulou-Stassinaki ${ }^{89}$, J. Stachel ${ }^{94}$, I. Stan ${ }^{62}$, P. Stankus ${ }^{85}$, G. Stefanek ${ }^{77}$, E. Stenlund ${ }^{34}$, G. Steyn ${ }^{65}$, J. H. Stiller ${ }^{94}$, D. Stocco ${ }^{113}$, P. Strmen ${ }^{39}$, A. A. P. Suaide ${ }^{120}$, T. Sugitate ${ }^{47}$, C. Suire ${ }^{51}$, M. Suleymanov ${ }^{16}$, M. Suljic ${ }^{26}$, R. Sultanov ${ }^{58}$, M. Šumbera ${ }^{84}$, A. Szabo ${ }^{39}$, A. Szanto de Toledo ${ }^{120, a}$, I. Szarka ${ }^{39}$, A. Szczepankiewicz ${ }^{36}$, M. Szymanski ${ }^{133}$,

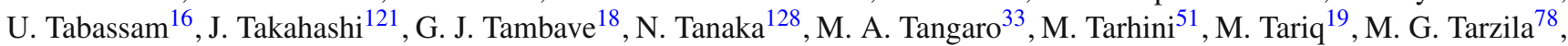
A. Tauro ${ }^{36}$, G. Tejeda Muñoz ${ }^{2}$, A. Telesca ${ }^{36}$, K. Terasaki ${ }^{127}$, C. Terrevoli ${ }^{30}$, B. Teyssier ${ }^{130}$, J. Thäder ${ }^{74}$, D. Thomas ${ }^{118}$, R. Tieulent ${ }^{130}$, A. R. Timmins ${ }^{122}$, A. Toia ${ }^{53}$, S. Trogolo ${ }^{27}$, G. Trombetta ${ }^{33}$, V. Trubnikov ${ }^{3}$, W. H. Trzaska ${ }^{123}$, T. Tsuji ${ }^{127}$, A. Tumkin ${ }^{99}$ ， R. Turrisi ${ }^{107}$, T. S. Tveter ${ }^{22}$, K. Ullaland ${ }^{18}$, A. Uras ${ }^{130}$ ， G. L. Usai ${ }^{25}$ ，A. Utrobicic ${ }^{129}$, M. Vajzer ${ }^{84}$, M. Vala ${ }^{59}$, L. Valencia Palomo ${ }^{70}$, S. Vallero ${ }^{27}$, J. Van Der Maarel ${ }^{57}$, J. W. Van Hoorne ${ }^{36}$, M. van Leeuwen ${ }^{57}$, T. Vanat ${ }^{84}$, P. Vande Vyvre ${ }^{36}$, D. Varga ${ }^{135}$, A. Vargas ${ }^{2}$, M. Vargyas ${ }^{123}$, R. Varma ${ }^{48}$, M. Vasileiou ${ }^{89}$, A. Vasiliev ${ }^{80}$, A. Vauthier ${ }^{71}$, V. Vechernin ${ }^{131}$, A. M. Veen ${ }^{57}$, M. Veldhoen ${ }^{57}$, A. Velure ${ }^{18}$, M. Venaruzzo ${ }^{73}$, E. Vercellin ${ }^{27}$, S. Vergara Limón ${ }^{2}$, R. Vernet ${ }^{8}$, M. Verweij ${ }^{134}$, L. Vickovic ${ }^{116}$, G. Viesti ${ }^{30, a}$, J. Viinikainen ${ }^{123}$, Z. Vilakazi ${ }^{126}$, O. Villalobos Baillie ${ }^{101}$, A. Villatoro Tello ${ }^{2}$, A. Vinogradov ${ }^{80}$, L. Vinogradov ${ }^{131}$, Y. Vinogradov ${ }^{99, a}$, T. Virgili ${ }^{31}$, V. Vislavicius ${ }^{34}$, Y. P. Viyogi ${ }^{132}$, A. Vodopyanov ${ }^{66}$, M. A. Völkl ${ }^{94}$, K. Voloshin ${ }^{58}$, S. A. Voloshin ${ }^{134}$, G. Volpe ${ }^{33}$, B. von Haller $^{36}$, I. Vorobyev $^{37,93}$, D. Vranic ${ }^{36,97}$, J. Vrlákováa ${ }^{41}$, B. Vulpescu ${ }^{70}$, B. Wagner ${ }^{18}$, J. Wagner ${ }^{97}$, H. Wang ${ }^{57}$, M. Wang ${ }^{7,113}$, D. Watanabe ${ }^{128}$, Y. Watanabe ${ }^{127}$, M. Weber ${ }^{36,112}$, S. G. Weber ${ }^{97}$, D. F. Weiser ${ }^{94}$, J. P. Wessels ${ }^{54}$, U. Westerhoff ${ }^{54}$, A. M. Whitehead ${ }^{90}$, J. Wiechula ${ }^{35}$, J. Wikne ${ }^{22}$, G. Wilk ${ }^{77}$, J. Wilkinson ${ }^{94}$, M. C. S. Williams ${ }^{104}$, B. Windelband ${ }^{94}$, M. Winn ${ }^{94}$, H. Yang ${ }^{57}$, P. Yang ${ }^{7}$, S. Yano ${ }^{47}$, C. Yasar ${ }^{69}$, Z. Yin ${ }^{7}$, H. Yokoyama ${ }^{128}$, I.-K. Yoo ${ }^{96}$, J. H. Yoon ${ }^{50}$, V. Yurchenko ${ }^{3}$, I. Yushmanov ${ }^{80}$, A. Zaborowska ${ }^{133}$, V. Zaccolo ${ }^{81}$, A. Zaman ${ }^{16}$, C. Zampolli ${ }^{36,104}$, H. J. C. Zanoli120, S. Zaporozhets ${ }^{66}$, N. Zardoshti101, A. Zarochentsev ${ }^{131}$, P. Závada ${ }^{60}$, N. Zaviyalov ${ }^{99}$, H. Zbroszczyk ${ }^{133}$, I. S. Zgura ${ }^{62}$, M. Zhalov ${ }^{86}$, H. Zhang ${ }^{18}$, X. Zhang ${ }^{74}$, Y. Zhang ${ }^{7}$, C. Zhang ${ }^{57}$, Z. Zhang ${ }^{7}$, C. Zhao ${ }^{22}$, N. Zhigareva ${ }^{58}$, D. Zhou ${ }^{7}$, Y. Zhou ${ }^{81}$, Z. Zhou ${ }^{18}$, H. Zhu ${ }^{18}$, J. Zhu ${ }^{7}{ }^{113}$, A. Zichichi ${ }^{12,28}$, A. Zimmermann ${ }^{94}$, M. B. Zimmermann ${ }^{36,54}$, G. Zinovjev ${ }^{3}$, M. Zyzak ${ }^{43}$

${ }^{1}$ A.I. Alikhanyan National Science Laboratory (Yerevan Physics Institute) Foundation, Yerevan, Armenia

2 Benemérita Universidad Autónoma de Puebla, Puebla, Mexico

${ }^{3}$ Bogolyubov Institute for Theoretical Physics, Kiev, Ukraine

${ }^{4}$ Department of Physics, Centre for Astroparticle Physics and Space Science (CAPSS), Bose Institute, Kolkata, India 
${ }^{5}$ Budker Institute for Nuclear Physics, Novosibirsk, Russia

${ }^{6}$ California Polytechnic State University, San Luis Obispo, CA, USA

${ }^{7}$ Central China Normal University, Wuhan, China

${ }^{8}$ Centre de Calcul de l'IN2P3, Villeurbanne, France

${ }^{9}$ Centro de Aplicaciones Tecnológicas y Desarrollo Nuclear (CEADEN), Havana, Cuba

${ }^{10}$ Centro de Investigaciones Energéticas Medioambientales y Tecnológicas (CIEMAT), Madrid, Spain

${ }^{11}$ Centro de Investigación y de Estudios Avanzados (CINVESTAV), Mexico City, Mérida, Mexico

${ }^{12}$ Centro Fermi-Museo Storico della Fisica e Centro Studi e Ricerche "Enrico Fermi”, Rome, Italy

${ }^{13}$ Chicago State University, Chicago, IL, USA

${ }^{14}$ China Institute of Atomic Energy, Beijing, China

${ }^{15}$ Commissariat à l'Energie Atomique, IRFU, Saclay, France

${ }^{16}$ COMSATS Institute of Information Technology (CIIT), Islamabad, Pakistan

${ }^{17}$ Departamento de Física de Partículas and IGFAE, Universidad de Santiago de Compostela, Santiago de Compostela, Spain

${ }^{18}$ Department of Physics and Technology, University of Bergen, Mons, Norway

${ }^{19}$ Department of Physics, Aligarh Muslim University, Aligarh, India

${ }^{20}$ Department of Physics, Ohio State University, Columbus, OH, USA

${ }^{21}$ Department of Physics, Sejong University, Seoul, South Korea

${ }^{22}$ Department of Physics, University of Oslo, Oslo, Norway

${ }^{23}$ Dipartimento di Elettrotecnica ed Elettronica del Politecnico, Bari, Italy

${ }^{24}$ Dipartimento di Fisica dell'Università 'La Sapienza' and Sezione INFN, Rome, Italy

${ }^{25}$ Dipartimento di Fisica dell'Università and Sezione INFN, Cagliari, Italy

${ }^{26}$ Dipartimento di Fisica dell'Università and Sezione INFN, Trieste, Italy

${ }^{27}$ Dipartimento di Fisica dell'Università and Sezione INFN, Turin, Italy

${ }^{28}$ Dipartimento di Fisica e Astronomia dell'Università and Sezione INFN, Bologna, Italy

${ }^{29}$ Dipartimento di Fisica e Astronomia dell'Università and Sezione INFN, Catania, Italy

${ }^{30}$ Dipartimento di Fisica e Astronomia dell'Università and Sezione INFN, Padua, Italy

${ }^{31}$ Dipartimento di Fisica 'E.R. Caianiello' dell'Università and Gruppo Collegato INFN, Salerno, Italy

${ }^{32}$ Dipartimento di Scienze e Innovazione Tecnologica dell'Università del Piemonte Orientale and Gruppo Collegato INFN, Alessandria, Italy

${ }^{33}$ Dipartimento Interateneo di Fisica 'M. Merlin' and Sezione INFN, Bari, Italy

${ }^{34}$ Division of Experimental High Energy Physics, University of Lund, Lund, Sweden

${ }^{35}$ Eberhard Karls Universität Tübingen, Tübingen, Germany

${ }^{36}$ European Organization for Nuclear Research (CERN), Geneva, Switzerland

${ }^{37}$ Excellence Cluster Universe, Technische Universität München, Munich, Germany

${ }^{38}$ Faculty of Engineering, Bergen University College, Mons, Norway

${ }^{39}$ Faculty of Mathematics, Physics and Informatics, Comenius University, Bratislava, Slovakia

${ }^{40}$ Faculty of Nuclear Sciences and Physical Engineering, Czech Technical University in Prague, Prague, Czech Republic

${ }^{41}$ Faculty of Science, P.J. Šafárik University, Kosice, Slovakia

${ }^{42}$ Faculty of Technology, Buskerud and Vestfold University College, Vestfold, Norway

${ }^{43}$ Frankfurt Institute for Advanced Studies, Johann Wolfgang Goethe-Universität Frankfurt, Frankfurt, Germany

${ }^{44}$ Gangneung-Wonju National University, Gangneung, South Korea

${ }^{45}$ Department of Physics, Gauhati University, Guwahati, India

${ }^{46}$ Helsinki Institute of Physics (HIP), Helsinki, Finland

${ }^{47}$ Hiroshima University, Hiroshima, Japan

${ }^{48}$ Indian Institute of Technology Bombay (IIT), Mumbai, India

${ }^{49}$ Indian Institute of Technology Indore (IITI), Indore, India

${ }^{50}$ Inha University, Inchon, South Korea

${ }^{51}$ Institut de Physique Nucléaire d'Orsay (IPNO), Université Paris-Sud, CNRS-IN2P3, Orsay, France

${ }^{52}$ Institut für Informatik, Johann Wolfgang Goethe-Universität Frankfurt, Frankfurt, Germany

${ }^{53}$ Institut für Kernphysik, Johann Wolfgang Goethe-Universität Frankfurt, Frankfurt, Germany

${ }^{54}$ Institut für Kernphysik, Westfälische Wilhelms-Universität Münster, Münster, Germany

${ }^{55}$ Institut Pluridisciplinaire Hubert Curien (IPHC), Université de Strasbourg, CNRS-IN2P3, Strasbourg, France 
${ }^{56}$ Institute for Nuclear Research, Academy of Sciences, Moscow, Russia

${ }^{57}$ Institute for Subatomic Physics of Utrecht University, Utrecht, The Netherlands

${ }^{58}$ Institute for Theoretical and Experimental Physics, Moscow, Russia

${ }^{59}$ Institute of Experimental Physics, Slovak Academy of Sciences, Kosice, Slovakia

${ }^{60}$ Institute of Physics, Academy of Sciences of the Czech Republic, Prague, Czech Republic

${ }^{61}$ Institute of Physics, Bhubaneswar, India

${ }^{62}$ Institute of Space Science (ISS), Bucharest, Romania

${ }^{63}$ Instituto de Ciencias Nucleares, Universidad Nacional Autónoma de México, Mexico City, Mexico

${ }^{64}$ Instituto de Física, Universidad Nacional Autónoma de México, Mexico City, Mexico

65 iThemba LABS, National Research Foundation, Somerset West, South Africa

${ }^{66}$ Joint Institute for Nuclear Research (JINR), Dubna, Russia

${ }^{67}$ Konkuk University, Seoul, South Korea

${ }^{68}$ Korea Institute of Science and Technology Information, Taejon, South Korea

${ }^{69}$ KTO Karatay University, Konya, Turkey

${ }^{70}$ Laboratoire de Physique Corpusculaire (LPC), Clermont Université, Université Blaise Pascal, CNRS-IN2P3, Clermont-Ferrand, France

${ }^{71}$ Laboratoire de Physique Subatomique et de Cosmologie, Université Grenoble-Alpes, CNRS-IN2P3, Grenoble, France

${ }^{72}$ Laboratori Nazionali di Frascati, INFN, Frascati, Italy

${ }^{73}$ Laboratori Nazionali di Legnaro, INFN, Legnaro, Italy

${ }^{74}$ Lawrence Berkeley National Laboratory, Berkeley, CA, USA

${ }^{75}$ Moscow Engineering Physics Institute, Moscow, Russia

${ }^{76}$ Nagasaki Institute of Applied Science, Nagasaki, Japan

77 National Centre for Nuclear Studies, Warsaw, Poland

78 National Institute for Physics and Nuclear Engineering, Bucharest, Romania

${ }^{79}$ National Institute of Science Education and Research, Bhubaneswar, India

${ }^{80}$ National Research Centre Kurchatov Institute, Moscow, Russia

${ }^{81}$ Niels Bohr Institute, University of Copenhagen, Copenhagen, Denmark

${ }^{82}$ Nikhef, Nationaal instituut voor subatomaire fysica, Amsterdam, The Netherlands

${ }^{83}$ Nuclear Physics Group, STFC Daresbury Laboratory, Daresbury, UK

${ }^{84}$ Nuclear Physics Institute, Academy of Sciences of the Czech Republic, Řež u Prahy, Czech Republic

${ }^{85}$ Oak Ridge National Laboratory, Oak Ridge, TN, USA

${ }^{86}$ Petersburg Nuclear Physics Institute, Gatchina, Russia

${ }^{87}$ Physics Department, Creighton University, Omaha, NE, USA

${ }^{88}$ Physics Department, Panjab University, Chandigarh, India

${ }^{89}$ Physics Department, University of Athens, Athens, Greece

${ }^{90}$ Physics Department, University of Cape Town, Cape Town, South Africa

${ }^{91}$ Physics Department, University of Jammu, Jammu, India

${ }^{92}$ Physics Department, University of Rajasthan, Jaipur, India

93 Physik Department, Technische Universität München, Munich, Germany

${ }^{94}$ Physikalisches Institut, Ruprecht-Karls-Universität Heidelberg, Heidelberg, Germany

${ }^{95}$ Purdue University, West Lafayette, IN, USA

${ }^{96}$ Pusan National University, Pusan, South Korea

${ }^{97}$ Research Division and ExtreMe Matter Institute EMMI, GSI Helmholtzzentrum für Schwerionenforschung, Darmstadt, Germany

${ }^{98}$ Rudjer Bošković Institute, Zagreb, Croatia

${ }^{99}$ Russian Federal Nuclear Center (VNIIEF), Sarov, Russia

${ }^{100}$ Saha Institute of Nuclear Physics, Kolkata, India

101 School of Physics and Astronomy, University of Birmingham, Birmingham, UK

102 Sección Física, Departamento de Ciencias, Pontificia Universidad Católica del Perú, Lima, Peru

${ }^{103}$ Sezione INFN, Bari, Italy

${ }^{104}$ Sezione INFN, Bologna, Italy

105 Sezione INFN, Cagliari, Italy

${ }^{106}$ Sezione INFN, Catania, Italy 
107 Sezione INFN, Padua, Italy

108 Sezione INFN, Rome, Italy

${ }^{109}$ Sezione INFN, Trieste, Italy

110 Sezione INFN, Turin, Italy

${ }^{111}$ SSC IHEP of NRC Kurchatov institute, Protvino, Russia

${ }^{112}$ Stefan Meyer Institut für Subatomare Physik (SMI), Vienna, Austria

${ }^{113}$ SUBATECH, Ecole des Mines de Nantes, Université de Nantes, CNRS-IN2P3, Nantes, France

${ }^{114}$ Suranaree University of Technology, Nakhon Ratchasima, Thailand

115 Technical University of Košice, Kosice, Slovakia

116 Technical University of Split FESB, Split, Croatia

117 The Henryk Niewodniczanski Institute of Nuclear Physics, Polish Academy of Sciences, Kraków, Poland

118 Physics Department, The University of Texas at Austin, Austin, TX, USA

${ }^{119}$ Universidad Autónoma de Sinaloa, Culiacán, Mexico

${ }^{120}$ Universidade de São Paulo (USP), São Paulo, Brazil

121 Universidade Estadual de Campinas (UNICAMP), Campinas, Brazil

122 University of Houston, Houston, TX, USA

${ }^{123}$ University of Jyväskylä, Jyväskylä, Finland

${ }^{124}$ University of Liverpool, Liverpool, UK

125 University of Tennessee, Knoxville, TN, USA

${ }^{126}$ University of the Witwatersrand, Johannesburg, South Africa

${ }^{127}$ University of Tokyo, Tokyo, Japan

128 University of Tsukuba, Tsukuba, Japan

${ }^{129}$ University of Zagreb, Zagreb, Croatia

${ }^{130}$ Université de Lyon, Université Lyon 1, CNRS/IN2P3, IPN-Lyon, Villeurbanne, France

${ }^{131}$ V. Fock Institute for Physics, St. Petersburg State University, St. Petersburg, Russia

132 Variable Energy Cyclotron Centre, Kolkata, India

${ }^{133}$ Warsaw University of Technology, Warsaw, Poland

${ }^{134}$ Wayne State University, Detroit, MI, USA

${ }^{135}$ Wigner Research Centre for Physics, Hungarian Academy of Sciences, Budapest, Hungary

136 Yale University, New Haven, CT, USA

137 Yonsei University, Seoul, South Korea

${ }^{138}$ Zentrum für Technologietransfer und Telekommunikation (ZTT), Fachhochschule Worms, Worms, Germany

a Deceased

${ }^{\mathrm{b}}$ Also at: Georgia State University, Atlanta, GA, USA

${ }^{c}$ Also at Department of Applied Physics, Aligarh Muslim University, Aligarh, India

d Also at: M.V. Lomonosov Moscow State University, D.V. Skobeltsyn Institute of Nuclear Physics, Moscow, Russia 